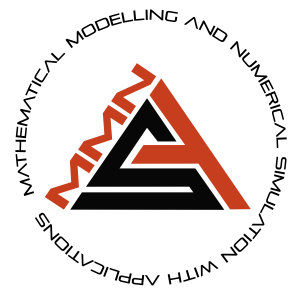

\title{
The Hermite-Hadamard type inequality and its estimations via generalized convex functions of Raina type
}

\author{
Muhammad Tariq 1,*,§, Hijaz Ahmad॰2,§ and Soubhagya Kumar \\ Sahoo $3, \S$
}

${ }^{1}$ Department of Basic Sciences and Related Studies, Mehran University of Engineering and Technology, Jamshoro 76062, Pakistan, ${ }^{2}$ Section of Mathematics, International Telematic University Uninettuno, Corso Vittorio Emanuele II, 39, 00186 Roma Italy, ${ }^{3}$ Department of Mathematics, Institute of Technical Education and Research, Siksha O Anusandhan University, Bhubaneswar 751030, Odisha, India

* Corresponding Author

\$captaintariq2187@gmail.com (Muhammad Tariq); hijaz555@gmail.com (Hijaz Ahmad); soubhagyalulu@gmail.com (Soubhagya Kumar Sahoo)

\begin{abstract}
The theory of convexity plays an important role in various branches of science and engineering. The main objective of this work is to introduce the idea of a generalized convex function by unifying s-type $\mathfrak{m}$-convex function and Raina type function. In addition, some beautiful algebraic properties and examples are discussed. Applying this new definition, we explore a new sort of Hermite-Hadamard inequality. Furthermore, to enhance the paper we investigate several new estimations of Hermite-Hadamard type inequality. The concepts and tools of this paper may invigorate and revitalize for additional research in this mesmerizing and absorbing field of mathematics.
\end{abstract}

Key words: Convex function; m-convex function; s-type convex function; Hölder's inequality; improved power-mean integral inequality

AMS 2020 Classification: 26A51; 26A33; 26D07; 26D10; 26D15

\section{Introduction}

The expression "convexity" is the main, intriguing, regular, and principal documentations in mathematical analysis. For the first time, it was utilized generally in a book by Hardy, Little, and Polya (see [1]). As of late, the hypothesis of convexity assumes an exceptionally entrancing and astonishing part in the realm of science, hence anyone working, especially in the field of inequalities cannot ignore its importance and significance. Numerous analysts consistently attempt to utilize novel thoughts for the pleasure and beautification of convexity theory. This hypothesis gives us fascinating and amazing mathematical strategies to tackle and to take care of a great deal of the issue which emerges in pure and applied sciences. During the last few decades, numerous scientists specially mathematicians have added to the advancement of the theory of convex analysis in different directions. For the attention of the readers, we encourage the references $[2,3,4,5,6,7]$ to see.

The theory of inequality is one of the most important aspects in many branches of mathematics such as functional analysis, theory of differential and integral equations, probability theory, mechanics, and other sciences. In this manner, the hypothesis of inequalities might be viewed as an autonomous field of mathematical analysis. As of late, the idea of convex analysis and the 
concept of inequality have been generalized, improved and extended in many directions. The relationship between these two fields has roused numerous scientists specially mathematicians because of its broad applications. For some related papers on convexity, see the references $[8,9,10,11]$.

As everybody knows, there exists a class of numerical models depicted by differential equations, for example, Malthus population model. In any case, a great deal of a differential equation does not have a specific arrangement. Under this case, integral inequalities are critical for researching the boundedness, stability, asymptotic behavior of solutions to differential equations.

Motivated by the ongoing research activities, the aim of this paper is to introduce a new class of a convex function, called a generalized $s$-type $\mathfrak{m}$-convex function of Raina type. Next, we explore some of its algebraic properties and examples. As main results, a new version of Hermite-Hadamard inequality and its refinements in support of the new definition are presented.

\section{Preliminaries}

Definition 1 [3] Let $\checkmark: I \rightarrow \mathbb{R}$ be a real valued function. A function $\checkmark$ is said to be convex, if

$$
\curlyvee\left(b a_{1}+(1-b) \mathfrak{a}_{2}\right) \leq b \curlyvee\left(\mathfrak{a}_{1}\right)+(1-b) \Upsilon\left(\mathfrak{a}_{2}\right)
$$

holds for all $\mathfrak{a}_{1}, \mathfrak{a}_{2} \in I$ and $b \in[0,1]$.

The most important inequality concerning convex functions is Hermite-Hadamard inequality [12] given as:

Theorem 1 If $\checkmark:\left[\mathfrak{a}_{1}, \mathfrak{a}_{2}\right] \rightarrow \mathbb{R}$ is a convex function, then

$$
\Upsilon\left(\frac{\mathfrak{a}_{1}+\mathfrak{a}_{2}}{2}\right) \leq \frac{1}{\mathfrak{a}_{2}-\mathfrak{a}_{1}} \int_{\mathfrak{a}_{1}}^{\mathfrak{a}_{2}} \Upsilon(x) d x \leq \frac{\Upsilon\left(\mathfrak{a}_{1}\right)+\Upsilon\left(\mathfrak{a}_{2}\right)}{2} .
$$

Since its discovery, many researchers have presented various generalizations and improvements with reference to different types of generalized convex functions like $s$ - convex functions, $m$ - convex functions, Harmonically convex functions, log-convex functions, exponentially convex functions, and many more. This inequality along with inequalities such as Ostrowski inequality, Simpson inequality, Bullen type inequality, Opial type inequality, and Mercer type inequality have accumulated a lot of attention among mathematicians due to their widespread view and applications in the field of mathematical analysis. In 2005, Raina [13] introduced a new class of function defined formally by

$$
\mathcal{F}_{\rho, \lambda}^{\sigma}(z)=\mathcal{F}_{\rho, \lambda}^{\sigma(0), \sigma(1), \ldots}(z)=\sum_{k=0}^{+\infty} \frac{\sigma(k)}{\Gamma(\rho k+\lambda)} z^{k}
$$

where $\sigma=(\sigma(0), \ldots, \sigma(k), \ldots)$ and $\rho, \lambda>0,|z|<R$. The above class of functions is a generalization of the classical Mittag-Leffler function and the Kummer function.

Cortez $[14,15]$ established a new class of set and function involving the Raina's function, which is said to be a generalized convex set and a convex function.

Definition $2[15]$ Let $\sigma=(\sigma(0), \ldots, \sigma(k), \ldots)$ and $\rho, \lambda>0$. A set $X \neq \emptyset$ is said to be generalized convex, if

$$
\mathfrak{a}_{2}+b \mathcal{F}_{\rho, \lambda}^{\sigma}\left(\mathfrak{a}_{1}-\mathfrak{a}_{2}\right) \in X,
$$

for all $\mathfrak{a}_{1}, \mathfrak{a}_{2} \in X$ and $b \in[0,1]$.

Definition 3 [15] Let $\sigma$ denote a bounded sequence, then $\sigma=(\sigma(0), \ldots, \sigma(k), \ldots)$ and $\rho, \lambda>0$. If $\curlyvee: X \rightarrow \mathbb{R}$ satisfies the following inequality

$$
\curlyvee\left(\mathfrak{a}_{2}+b \mathcal{F}_{\rho, \lambda}^{\sigma}\left(\mathfrak{a}_{1}-\mathfrak{a}_{2}\right)\right) \leq b \curlyvee\left(\mathfrak{a}_{1}\right)+(1-b) \Upsilon\left(\mathfrak{a}_{2}\right)
$$

for all $\mathfrak{a}_{1}, \mathfrak{a}_{2} \in X$, where $\mathfrak{a}_{1}<\mathfrak{a}_{2}$ and $b \in[0,1]$, then $\Upsilon$ is called a generalized convex function.

Remark 1 We have $\mathcal{F}_{\rho, \lambda}^{\sigma}\left(\mathfrak{a}_{1}-\mathfrak{a}_{2}\right)=\mathfrak{a}_{1}-\mathfrak{a}_{2}>0$, and so we obtain Definition 1 .

Condition A: Let $X \subseteq \mathbb{R}$ be an open generalized convex subset with respect to (w.r.t.) $\mathcal{F}_{\rho, \lambda}^{\sigma}(\cdot)$. For any $\mathfrak{a}_{1}, \mathfrak{a}_{2} \in X$ and $b \in[0,1]$,

$$
\begin{gathered}
\mathcal{F}_{\rho, \lambda}^{\sigma}\left(\mathfrak{a}_{2}-\left(\mathfrak{a}_{2}+b \mathcal{F}_{\rho, \lambda}^{\sigma}\left(\mathfrak{a}_{1}-\mathfrak{a}_{2}\right)\right)\right)=-b \mathcal{F}_{\rho, \lambda}^{\sigma}\left(\mathfrak{a}_{1}-\mathfrak{a}_{2}\right), \\
\mathcal{F}_{\rho, \lambda}^{\sigma}\left(\mathfrak{a}_{1}-\left(\mathfrak{a}_{2}+b \mathcal{F}_{\rho, \lambda}^{\sigma}\left(\mathfrak{a}_{1}-\mathfrak{a}_{2}\right)\right)\right)=(1-b) \mathcal{F}_{\rho, \lambda}^{\sigma}\left(\mathfrak{a}_{1}-\mathfrak{a}_{2}\right) .
\end{gathered}
$$

Note that, for every $\mathfrak{a}_{1}, \mathfrak{a}_{2} \in X$ and for all $b_{1}, b_{2} \in[0,1]$, using Condition A, we have

$$
\mathcal{F}_{\rho, \lambda}^{\sigma}\left(\mathfrak{a}_{2}+b_{2} \mathcal{F}_{\rho, \lambda}^{\sigma}\left(\mathfrak{a}_{1}-\mathfrak{a}_{2}\right)-\left(\mathfrak{a}_{2}+b_{1} \mathcal{F}_{\rho, \lambda}^{\sigma}\left(\mathfrak{a}_{1}-\mathfrak{a}_{2}\right)\right)\right)=\left(b_{2}-b_{1}\right) \mathcal{F}_{\rho, \lambda}^{\sigma}\left(\mathfrak{a}_{1}-\mathfrak{a}_{2}\right) .
$$


Definition 4 [16] A non-negative function $\checkmark: \mathbb{A} \rightarrow \mathbb{R}$ is said to be an s-type convex function if the following inequality for $\mathfrak{a}_{1}, \mathfrak{a}_{2} \in \mathbb{A}$, $s \in[0,1]$ and $b \in[0,1]$ holds true:

$$
\curlyvee\left(b a_{1}+(1-b) \mathfrak{a}_{2}\right) \leq[1-(s(1-b))] \curlyvee\left(\mathfrak{a}_{1}\right)+[1-s b] \curlyvee\left(\mathfrak{a}_{2}\right)
$$

Definition 5 [3] An inequality of the form

$$
\left(\curlyvee\left(\mathfrak{a}_{1}\right)-\curlyvee\left(\mathfrak{a}_{2}\right)\right)\left(\Psi\left(\mathfrak{a}_{1}\right)-\Psi\left(\mathfrak{a}_{2}\right)\right) \geq 0, \quad \forall \mathfrak{a}_{1}, \mathfrak{a}_{2} \in \mathbb{R}
$$

is said to be similarly ordered.

Inspired and excited by the ongoing research activities, the construction of this paper is marked as follows. First of all, in section 3, we discuss the new definition of the generalized $s$-type $(m)$-convex function of Raina type and its algebraic properties. In section 4 , on the basis of a new identity, we attain the refinements of Hermite-Hadamard type inequality employing the new definition.

\section{Generalized Preinvex function and its algebraic properties}

In this section, we present the definition of a new class of convex functions called generalized $s$-type $m$-convex function of Raina type and also discuss its algebraic properties.

Definition 6 Let $\mathbb{X}$ be a nonempty generalized convex set w.r.t. $\mathcal{F}_{\rho, \lambda}^{\sigma}: \mathbb{X} \times \mathbb{X} \rightarrow \mathbb{R}$.Then the nonnegative function $\checkmark: \mathbb{X} \rightarrow \mathbb{R}$ is said to be a generalized s-type $\mathfrak{m}$-convex function of Raina type for fixed $\mathfrak{m} \mathfrak{m} \in(0,1] \times(0,1]$, if

$$
\curlyvee\left(\mathfrak{a}_{2}+b \mathcal{F}_{\rho, \lambda}^{\sigma}\left(\mathfrak{a}_{1}-\mathfrak{a}_{2}\right)\right) \leq(1-s b) \Upsilon\left(\mathfrak{a}_{2}\right)+(1-(s(1-b))) \mathfrak{m} \Upsilon\left(\frac{\mathfrak{a}_{1}}{\mathfrak{m}}\right)
$$

holds for every $\mathfrak{a}_{1}, \mathfrak{a}_{2} \in \mathbb{X}, \sigma=(\sigma(0), \ldots, \sigma(k), \ldots), \rho, \lambda>0, s \in[0,1]$, and $b \in[0,1]$.

Remark 2 (i) Taking $s=\mathfrak{m}=1$ in Definition 6, we attain the definition of a generalized convex function of Raina type which was explored by Cortez [14, 15].

(ii) Taking $\mathfrak{m}=1$ and $\mathcal{F}_{\rho, \lambda}^{\sigma}\left(\mathfrak{a}_{1}-\mathfrak{a}_{2}\right)=\mathfrak{a}_{1}-\mathfrak{a}_{2}$ in Definition 6, we attain the definition of s-type convex function which was given by İscan et al. [16].

(iii) Taking $s=\mathfrak{m}=1$ and $\mathcal{F}_{\rho, \lambda}^{\sigma}\left(\mathfrak{a}_{1}-\mathfrak{a}_{2}\right)=\mathfrak{a}_{1}-\mathfrak{a}_{2}$ in Definition 6, we obtain the definition, namely a convex function which was investigated by Niculescu et al. [3].

Lemma 1 The following inequalities

$$
b \leq(1-(s(1-b))) \text { and } 1-b \leq 1-s b
$$

hold, for all $b \in[0,1], \mathfrak{m} \in(0,1]$ and $s \in[0,1]$.

Proof The rest of the proof is clearly seen.

Proposition 1 Every nonnegative generalized $\mathfrak{m}$-convex function of Raina type is a generalized s-type $\mathfrak{m}$-convex function of Raina type for $s \in[0,1], \mathfrak{m} \in(0,1]$ and $b \in[0,1]$.

Proof By using Lemma 1 and the definition of a generalized $\mathfrak{m}$-convex function of Raina type for $s \in[0,1], \mathfrak{m} \in(0,1]$ and $b \in[0,1]$, we have

$$
\begin{aligned}
& \curlyvee\left(\mathfrak{a}_{2}+b \mathcal{F}_{\rho, \lambda}^{\sigma}\left(\mathfrak{a}_{1}-\mathfrak{a}_{2}\right)\right) \leq(1-b) \curlyvee\left(\mathfrak{a}_{2}\right)+\mathfrak{m} b \curlyvee\left(\frac{\mathfrak{a}_{1}}{\mathfrak{m}}\right) \\
& \leq(1-s b) \curlyvee\left(\mathfrak{a}_{2}\right)+(1-(s(1-b))) \mathfrak{m} \Upsilon\left(\frac{\mathfrak{a}_{1}}{\mathfrak{m}}\right) .
\end{aligned}
$$

Proposition 2 Every non-negative generalized s-type $\mathfrak{m}$-convex function of Raina type for $s \in[0,1], \mathfrak{m} \in(0,1], n \in \mathbb{N}$ and $b \in[0,1]$, is a generalized $(h, \mathfrak{m})$-convex function of Raina type with $h(b)=(1-(s(1-b)))$.

Proof Using the definition of a generalized $s$-type $\mathfrak{m}$-convex function of Raina type for $s \in[0,1], \mathfrak{m} \in(0,1]$ and $b \in[0,1]$ and in view of the condition $h(b)=(1-(s(1-b)))$, we have

$$
\begin{gathered}
\curlyvee\left(\mathfrak{a}_{2}+b \mathcal{F}_{\rho, \lambda}^{\sigma}\left(\mathfrak{a}_{1}-\mathfrak{a}_{2}\right)\right) \leq(1-s b) \curlyvee\left(\mathfrak{a}_{2}\right)+(1-(s(1-b))) \mathfrak{m} \Upsilon\left(\frac{\mathfrak{a}_{1}}{\mathfrak{m}}\right) \\
\leq h(1-b) \curlyvee\left(\mathfrak{a}_{2}\right)+h \mathfrak{m}(b) \curlyvee\left(\frac{\mathfrak{a}_{1}}{\mathfrak{m}}\right) .
\end{gathered}
$$

Now, we will investigate some algebraic properties of the new definition. 
Theorem 2 Let $\Upsilon, \Psi: \mathbb{A}=\left[\mathfrak{a}_{1}, \mathfrak{a}_{2}\right] \rightarrow \mathbb{R}$. If $\Upsilon, \Psi$ are two generalized s-type $\mathfrak{m}$-convex functions of Raina type w.r.t. the same $\mathcal{F}_{\rho, \lambda}^{\sigma}$, then

(i) The sum of $\Upsilon$ and $\Psi$ is again a generalized $s$-type $\mathfrak{m}$-convex function of Raina type w.r.t. $\mathcal{F}_{\rho, \lambda}^{\sigma}$.

(ii) The scalar multiplication of the function $\curlyvee$ is a generalized $s$-type $\mathfrak{m}$-convex function of Raina type w.r.t. $\mathcal{F}_{\rho, \lambda}^{\sigma}$.

Proof $(i)$ Let $\Upsilon, \Psi$ be generalized $s$-type $\mathfrak{m}$-convex functions of Raina type w.r.t. the $\mathcal{F}_{\rho, \lambda}^{\sigma}$, then for all $\mathfrak{a}_{1}, \mathfrak{a}_{2} \in \mathbb{X}, s \in[0,1]$, $\mathfrak{m} \in(0,1]$ and $b \in[0,1]$, we have

$$
\begin{aligned}
& (\Upsilon+\Psi)\left(\mathfrak{a}_{2}+b \mathcal{F}_{\rho, \lambda}^{\sigma}\left(\mathfrak{a}_{1}-\mathfrak{a}_{2}\right)\right) \\
& =\curlyvee\left(\mathfrak{a}_{2}+b \mathcal{F}_{\rho, \lambda}^{\sigma}\left(\mathfrak{a}_{1}-\mathfrak{a}_{2}\right)\right)+\Psi\left(\mathfrak{a}_{2}+b \mathcal{F}_{\rho, \lambda}^{\sigma}\left(\mathfrak{a}_{1}-\mathfrak{a}_{2}\right)\right) \\
& \leq(1-s b) \Upsilon\left(\mathfrak{a}_{2}\right)+(1-(s(1-b))) m \curlyvee\left(\frac{\mathfrak{a}_{1}}{\mathfrak{m}}\right) \\
& +(1-s b) \Psi\left(\mathfrak{a}_{2}\right)+(1-(s(1-b))) \mathfrak{m} \Psi\left(\frac{\mathfrak{a}_{1}}{\mathfrak{m}}\right) \\
& =(1-s b)\left[\curlyvee\left(\mathfrak{a}_{2}\right)+\Psi\left(\mathfrak{a}_{2}\right)\right]+(1-(s(1-b)))\left[\mathfrak{m} \Upsilon\left(\frac{\mathfrak{a}_{1}}{\mathfrak{m}}\right)+\mathfrak{m} \Psi\left(\frac{\mathfrak{a}_{1}}{\mathfrak{m}}\right)\right] \\
& =(1-s b)(\curlyvee+\Psi)\left(\mathfrak{a}_{2}\right)+(1-(s(1-b))) \mathfrak{m}(\Upsilon+\Psi)\left(\frac{\mathfrak{a}_{1}}{\mathfrak{m}}\right) .
\end{aligned}
$$

(ii) Let $\checkmark$ be a generalized $s$-type $\mathfrak{m}$-convex function of Raina type w.r.t. $\quad \mathcal{F}_{\rho, \lambda}^{\sigma}$, then for all $\mathfrak{a}_{1}, \mathfrak{a}_{2} \in \mathbb{A}, s \in[0,1], c \in \mathbb{R}(c \geq 0)$, $\mathfrak{m} \in(0,1]$ and $b \in[0,1]$, we have

$$
\begin{aligned}
& (c \curlyvee)\left(\mathfrak{a}_{2}+b \mathcal{F}_{\rho, \lambda}^{\sigma}\left(\mathfrak{a}_{1}-\mathfrak{a}_{2}\right)\right) \\
& \leq c\left[(1-s b) \Upsilon\left(\mathfrak{a}_{2}\right)+(1-(s(1-b))) \mathfrak{m} \Upsilon\left(\frac{\mathfrak{a}_{1}}{\mathfrak{m}}\right)\right] \\
& =(1-s b) c \curlyvee\left(\mathfrak{a}_{2}\right)+(1-(s(1-b))) c \mathfrak{m} \Upsilon\left(\frac{\mathfrak{a}_{1}}{\mathfrak{m}}\right) \\
& =(1-s b)(c \curlyvee)\left(\mathfrak{a}_{2}\right)+(1-(s(1-b))) \mathfrak{m}(c \curlyvee)\left(\frac{\mathfrak{a}_{1}}{\mathfrak{m}}\right) .
\end{aligned}
$$

It is the required proof.

Theorem 3 Let $\checkmark: \mathbb{A} \rightarrow \mathbb{Y}$ be a generalized s-type $\mathfrak{m}$-convex function of Raina type w.r.t. $\mathcal{F}_{\rho, \lambda}^{\sigma}$ and $\Psi: \mathbb{Y} \rightarrow \mathbb{R}$ be a non-decreasing function. Then the function $\Psi \circ \Upsilon$ is a generalized $s$-type $\mathfrak{m}$-convex function of Raina type w.r.t. $\mathcal{F}_{\rho, \lambda}^{\sigma}$ for $s \in[0,1], \mathfrak{m} \in(0,1]$ and $b \in[0,1]$.

Proof For all $\mathfrak{a}_{1}, \mathfrak{a}_{2} \in \mathbb{A}, s \in[0,1], \mathfrak{m} \in(0,1]$ and $b \in[0,1]$, we have

$$
\begin{aligned}
& (\Psi \circ \Upsilon)\left(\mathfrak{a}_{2}+b \mathcal{F}_{\rho, \lambda}^{\sigma}\left(\mathfrak{a}_{1}-\mathfrak{a}_{2}\right)\right) \\
& =\Psi\left(\curlyvee\left(\mathfrak{a}_{2}+b \mathcal{F}_{\rho, \lambda}^{\sigma}\left(\mathfrak{a}_{1}-\mathfrak{a}_{2}\right)\right)\right) \\
& \leq \Psi\left[(1-s b) \curlyvee\left(\mathfrak{a}_{2}\right)+(1-(s(1-b))) \mathfrak{m} \Upsilon\left(\frac{\mathfrak{a}_{1}}{\mathfrak{m}}\right)\right] \\
& \leq(1-s b) \Psi\left(\Upsilon\left(\mathfrak{a}_{2}\right)\right)+(1-(s(1-b))) \mathfrak{m} \Psi\left(\mathfrak{m} \Upsilon\left(\frac{\mathfrak{a}_{1}}{\mathfrak{m}}\right)\right) \\
& =(1-s b)(\Psi \circ \Upsilon)\left(\mathfrak{a}_{2}\right)+(1-(s(1-b))) \mathfrak{m}^{2}(\Psi \circ \Upsilon)\left(\frac{\mathfrak{a}_{1}}{\mathfrak{m}}\right) .
\end{aligned}
$$

It is the required proof.

Remark 3 (i) If $n=s=1$ in Theorem 3, then

$$
(\Psi \circ \Upsilon)\left(\mathfrak{m a} \mathfrak{a}_{2}+b \eta\left(\mathfrak{a}_{1}, \mathfrak{a}_{2}, \mathfrak{m}\right)\right) \leq(1-b)(\Psi \circ \Upsilon)\left(\mathfrak{a}_{2}\right)+b \mathfrak{m}^{2}(\Psi \circ \Upsilon)\left(\frac{\mathfrak{a}_{1}}{\mathfrak{m}}\right)
$$

(i) If $s=\mathfrak{m}=1$ in Theorem 3 , then

$$
(\Psi \circ \Upsilon)\left(\mathfrak{a}_{2}+b \mathcal{F}_{\rho, \lambda}^{\sigma}\left(\mathfrak{a}_{1}-\mathfrak{a}_{2}\right)\right) \leq(1-b)(\Psi \circ \Upsilon)\left(\mathfrak{a}_{2}\right)+b(\Psi \circ \Upsilon)\left(\mathfrak{a}_{1}\right)
$$

(ii) If we put $\mathfrak{m}=1$ and $\eta\left(\mathfrak{a}_{1}, \mathfrak{a}_{2}, \mathfrak{m}\right)=\mathfrak{a}_{1}-\mathfrak{m} \mathfrak{a}_{2}$ in Theorem 3 , then

$$
(\Psi \circ \Upsilon)\left(b \mathfrak{a}_{1}+(1-b) \mathfrak{a}_{2}\right) \leq(1-s b)(\Psi \circ \Upsilon)\left(\mathfrak{a}_{2}\right)+\left(1-(s(1-b))(\Psi \circ \Upsilon)\left(\mathfrak{a}_{1}\right)\right.
$$

Theorem 4 Let $0<\mathfrak{a}_{1}<\mathfrak{a}_{2}, \Upsilon_{j}: \mathbb{A}=\left[\mathfrak{a}_{1}, \mathfrak{a}_{2}\right] \rightarrow[0,+\infty)$ be a class of generalized s-type $\mathfrak{m}$-convex functions of Raina type w.r.t. $\mathcal{F}_{\rho, \lambda}^{\sigma}$ and $\Upsilon(u)=\sup _{j} \Upsilon_{j}(u)$. Then $\Upsilon$ is a generalized s-type $\mathfrak{m}$-convex function of Raina type for $s \in[0,1], \mathfrak{m} \in(0,1]$ and $b \in[0,1]$, and $U=\left\{\tau \in\left[\mathfrak{a}_{1}, \mathfrak{a}_{2}\right]: \curlyvee\left(\tau_{i}\right)<\infty\right\}$ is an interval. 
Proof Let $\mathfrak{a}_{1}, \mathfrak{a}_{2} \in U, s \in[0,1], \mathfrak{m} \in(0,1]$ and $b \in[0,1]$, then

$$
\begin{aligned}
& \Upsilon\left(\mathfrak{a}_{2}+b \mathcal{F}_{\rho, \lambda}^{\sigma}\left(\mathfrak{a}_{1}-\mathfrak{a}_{2}\right)\right) \\
& =\sup _{j} \Upsilon_{j}\left(\mathfrak{a}_{2}+b \mathcal{F}_{\rho, \lambda}^{\sigma}\left(\mathfrak{a}_{1}-\mathfrak{a}_{2}\right)\right) \\
& \leq(1-s b) \sup _{j} \Upsilon_{j}\left(\mathfrak{a}_{2}\right)+(1-(s(1-b))) \mathfrak{m} \sup _{j} \Upsilon_{j}\left(\frac{\mathfrak{a}_{1}}{\mathfrak{m}}\right) \\
& =(1-s b) \Upsilon\left(\mathfrak{a}_{2}\right)+(1-(s(1-b))) \mathfrak{m} \Upsilon\left(\frac{\mathfrak{a}_{1}}{\mathfrak{m}}\right)<\infty \text {. }
\end{aligned}
$$

This is the required proof.

Theorem 5 If $\Upsilon_{i}: \mathbb{R}^{n} \rightarrow \mathbb{R}$ is a generalized $s$-type $\mathfrak{m}$-convex function of Raina type w.r.t. $\mathcal{F}_{\rho, \lambda}^{\sigma}$ for $s \in[0,1], \mathfrak{m} \in(0,1]$ and $b \in[0,1]$, then the set $\mathbb{M}=\left\{\tau \in \mathbb{R}: \Upsilon_{i}(\tau) \leq 0, i=1,2,3, \ldots, n\right\}$ is a generalized $\mathfrak{m}$-convex set.

Proof Since $\Upsilon_{i}(\tau),(i=1,2,3, \ldots, n)$ are generalized $s$-type $\mathfrak{m}$-convex functions of Raina type for $s \in[0,1], \mathfrak{m} \in(0,1]$ and $b \in[0,1]$, then for all $\mathfrak{a}_{1}, \mathfrak{a}_{2} \in \mathbb{R}^{n}$

$$
\Upsilon_{i}\left(\mathfrak{a}_{2}+b \mathcal{F}_{\rho, \lambda}^{\sigma}\left(\mathfrak{a}_{1}-\mathfrak{a}_{2}\right)\right) \leq(1-s b) \Upsilon\left(\mathfrak{a}_{2}\right)+(1-(s(1-b))) \mathfrak{m} \Upsilon\left(\frac{\mathfrak{a}_{1}}{\mathfrak{m}}\right)
$$

holds, where $i=1,2,3, \ldots, n$

When $\mathfrak{a}_{1}, \mathfrak{a}_{2} \in \mathbb{M}$, we know $\Upsilon_{i}\left(\mathfrak{a}_{1}\right) \leq 0$ and $\Upsilon_{i}\left(\mathfrak{a}_{2}\right) \leq 0$, from the above inequality, it yields that

$$
\Upsilon_{i}\left(\mathfrak{a}_{2}+b \mathcal{F}_{\rho, \lambda}^{\sigma}\left(\mathfrak{a}_{1}-\mathfrak{a}_{2}\right)\right) \leq 0, \quad i=1,2,3, \ldots, n \text {. }
$$

That is $\mathfrak{a}_{2}+b \mathcal{F}_{\rho, \lambda}^{\sigma}\left(\mathfrak{a}_{1}-\mathfrak{a}_{2}\right) \in \mathbb{M}$. Hence, $\mathbb{M}$ is a generalized $\mathfrak{m}$-convex set.

Theorem 6 If $\Upsilon: \mathbb{A} \subseteq \mathbb{R}^{n} \rightarrow \mathbb{R}$ is a generalized $s$-type $\mathfrak{m}$-convex function of Raina type w.r.t. $\mathcal{F}_{\rho, \lambda}^{\sigma}$ for $s \in[0,1], \mathfrak{m} \in(0,1]$ and $b \in[0,1]$, then the function $\Upsilon$ is also a generalized quasi $\mathfrak{m}$-convex function of Raina type on a generalized $\mathfrak{m}$-convex set of Raina type $\mathbb{A}$ w.r.t. $\mathcal{F}_{\rho, \lambda}^{\sigma}$.

Proof Since $\Upsilon$ is a generalized $s$-type $\mathfrak{m}$-convex function of Raina type w.r.t. $\mathcal{F}_{\rho, \lambda}^{\sigma}$ for $s \in[0,1], \mathfrak{m} \in(0,1]$ and $b \in[0,1]$, and we assume that $m \curlyvee\left(\frac{\mathfrak{a}_{1}}{\mathfrak{m}}\right) \leq \curlyvee\left(\mathfrak{a}_{2}\right)$, then for all $\mathfrak{a}_{1}, \mathfrak{a}_{2} \in \mathbb{A}$, we have

$$
\begin{aligned}
& \Upsilon\left(\mathfrak{a}_{2}+b \mathcal{F}_{\rho, \lambda}^{\sigma}\left(\mathfrak{a}_{1}-\mathfrak{a}_{2}\right)\right) \\
& \leq(1-s b) \Upsilon\left(\mathfrak{a}_{2}\right)+(1-(s(1-b))) \mathfrak{m} \Upsilon\left(\frac{\mathfrak{a}_{1}}{\mathfrak{m}}\right) \\
& \leq[(1-(s(1-b)))+(1-s b)] \curlyvee\left(\mathfrak{a}_{2}\right) \\
& \leq \Upsilon\left(\mathfrak{a}_{2}\right) .
\end{aligned}
$$

In the same manner, let $\curlyvee\left(\mathfrak{a}_{2}\right) \leq \mathfrak{m} \Upsilon\left(\frac{\mathfrak{a}_{1}}{\mathfrak{m}}\right)$, for all $\mathfrak{a}_{1}, \mathfrak{a}_{2} \in \mathbb{A}$. We can also get

$$
\curlyvee\left(\mathfrak{a}_{2}+b \mathcal{F}_{\rho, \lambda}^{\sigma}\left(\mathfrak{a}_{1}-\mathfrak{a}_{2}\right)\right) \leq \mathfrak{m} \Upsilon\left(\frac{\mathfrak{a}_{1}}{\mathfrak{m}}\right) .
$$

Consequently,

$$
\Upsilon\left(\mathfrak{a}_{2}+b \mathcal{F}_{\rho, \lambda}^{\sigma}\left(\mathfrak{a}_{1}-\mathfrak{a}_{2}\right)\right) \leq \max \left\{\curlyvee\left(\mathfrak{a}_{1}\right), \curlyvee\left(\mathfrak{a}_{2}\right)\right\} .
$$

That is, $\curlyvee: \mathbb{A} \subseteq \mathbb{R}^{n} \rightarrow \mathbb{R}$ is a generalized quasi $\mathfrak{m}$-convex function of Raina type on a generalized $\mathfrak{m}$-convex set of Raina type $\mathbb{A}$ w.r.t. $\mathcal{F}_{\rho, \lambda}^{\sigma}$.

Theorem 7 If $\checkmark: \mathbb{R}_{\circ} \rightarrow \mathbb{R}_{\circ}$ is a generalized s-type $\mathfrak{m}$-convex function of Raina type w.r.t. $\quad \mathcal{F}_{0, \lambda}^{\sigma}: \mathbb{R}_{\circ} \times \mathbb{R}_{\circ} \times(0,1] \rightarrow \mathbb{R}_{\circ}$ for $s \in[0,1]$, $\mathfrak{m} \in(0,1]$ and $b \in[0,1]$. Assume that $\checkmark$ is monotone decreasing, $\mathcal{F}_{\rho, \lambda}^{\sigma}$ is monotone increasing regarding $\mathfrak{m}$ for fixed $\mathfrak{a}_{1}, \mathfrak{a}_{2} \in \mathbb{R}_{\circ}$ and $\mathfrak{m}_{1} \leq \mathfrak{m}_{2} \quad\left(\mathfrak{m}_{1}, \mathfrak{m}_{2} \in(0,1]\right)$. If $\curlyvee$ is an $s$-type $\mathfrak{m}_{1}$-preinvex function on $\mathbb{R}_{\circ}$ w.r.t. $\mathcal{F}_{\rho, \lambda}^{\sigma}$, then $\curlyvee$ is an s-type $\mathfrak{m}_{2}$-preinvex function on $\mathbb{R}_{\circ}$ w.r.t. $\mathcal{F}_{\rho, \lambda}^{\sigma}$.

Proof Since $\Upsilon$ is a generalized s-type $m_{1}$-convex function of Raina type, for all $\mathfrak{a}_{1}, \mathfrak{a}_{2} \in \mathbb{R}_{\circ}$

$$
\curlyvee\left(\mathfrak{a}_{2}+b \mathcal{F}_{\rho, \lambda}^{\sigma}\left(\mathfrak{a}_{1}-\mathfrak{a}_{2}\right)\right) \leq(1-s b) \Upsilon\left(\mathfrak{a}_{2}\right)+(1-(s(1-b))) \mathfrak{m}_{1} \curlyvee\left(\frac{\mathfrak{a}_{1}}{\mathfrak{m}_{1}}\right) \text {. }
$$

Combining the monotone decreasing of the function $\checkmark$ with the monotone increasing of the mapping $\mathcal{F}_{\rho, \lambda}^{\sigma}$ regarding $\mathfrak{m}$ for fixed $\mathfrak{a}_{1}, \mathfrak{a}_{2} \in \mathbb{R}_{\circ}$ and $m_{1} \leq m_{2}$, it follows that

$$
\begin{aligned}
& (1-s b) \curlyvee\left(\mathfrak{a}_{2}\right)+(1-(s(1-b))) \mathfrak{m}_{1} \curlyvee\left(\frac{\mathfrak{a}_{1}}{\mathfrak{m}_{1}}\right) \\
& \leq(1-s b) \curlyvee\left(\mathfrak{a}_{2}\right)+(1-(s(1-b))) \mathfrak{m}_{2} \curlyvee\left(\frac{\mathfrak{a}_{1}}{\mathfrak{m}_{2}}\right) .
\end{aligned}
$$


Finally, we have

$$
\curlyvee\left(\mathfrak{a}_{2}+b \mathcal{F}_{\rho, \lambda}^{\sigma}\left(\mathfrak{a}_{1}-\mathfrak{a}_{2}\right)\right) \leq(1-s b) \curlyvee\left(\mathfrak{a}_{2}\right)+(1-(s(1-b))) m_{2} \curlyvee\left(\frac{\mathfrak{a}_{1}}{m_{2}}\right)
$$

Hence, $\checkmark$ is a generalized $s$-type $\mathfrak{m}_{2}$-convex function of Raina type on $\mathbb{R}_{\circ}$ w.r.t. $\eta$ for fixed $s \in[0,1]$ and $\mathfrak{m} \in[0,1]$, which ends the proof.

Theorem 8 Let $\Upsilon, \Psi: \mathbb{A}=\left[\mathfrak{a}_{1}, \mathfrak{a}_{2}\right] \rightarrow \mathbb{R}$. If $\Upsilon, \Psi$ are two generalized s-type $\mathfrak{m}$-convex functions of Raina type w.r.t. $\mathcal{F}_{\rho, \lambda}^{\sigma}$ and $\Upsilon$, $\Psi$ are similarly ordered functions and $[1-(s(1-b))]+[1-s b] \leq 1$, then the product $\curlyvee \Psi$ is a generalized $s$-type $m$-convex function of Raina type w.r.t. $\mathcal{F}_{\rho, \lambda}^{\sigma}$ for $s \in[0,1], \mathfrak{m} \in(0,1]$ and $b \in[0,1]$.

Proof Let $\Upsilon, \Psi$ be a generalized $s$-type $\mathfrak{m}$-convex function of Raina type w.r.t. same $\mathcal{F}_{\rho, \lambda}^{\sigma}, s \in[0,1], \mathfrak{m} \in(0,1]$ and $b \in[0,1]$, then

$$
\begin{aligned}
& \curlyvee\left(\mathfrak{a}_{2}+b \mathcal{F}_{\rho, \lambda}^{\sigma}\left(\mathfrak{a}_{1}-\mathfrak{a}_{2}\right)\right) \Psi\left(\mathfrak{a}_{2}+b \mathcal{F}_{\rho, \lambda}^{\sigma}\left(\mathfrak{a}_{1}-\mathfrak{a}_{2}\right)\right) \\
& \leq\left[(1-s b) \Upsilon\left(\mathfrak{a}_{2}\right)+(1-(s(1-b))) \mathfrak{m} \Upsilon\left(\frac{\mathfrak{a}_{1}}{\mathfrak{m}}\right)\right] \\
& \times\left[(1-s b) \Psi\left(\mathfrak{a}_{2}\right)+(1-(s(1-b))) \mathfrak{m} \Psi\left(\frac{\mathfrak{a}_{1}}{\mathfrak{m}}\right)\right] \\
& \leq(1-s b)^{2} \curlyvee\left(\mathfrak{a}_{2}\right) \Psi\left(\mathfrak{a}_{2}\right)+(1-(s(1-b)))^{2} \mathfrak{m}^{2} \curlyvee\left(\frac{\mathfrak{a}_{1}}{\mathfrak{m}}\right) \Psi\left(\frac{\mathfrak{a}_{1}}{\mathfrak{m}}\right) \\
& +\frac{1}{n^{2}}(1-(s(1-b)))(1-s b)\left[\mathfrak{m} \Upsilon\left(\frac{\mathfrak{a}_{1}}{\mathfrak{m}}\right) \Psi\left(\mathfrak{a}_{2}\right)+\curlyvee\left(\mathfrak{a}_{2}\right) \mathfrak{m} \Psi\left(\frac{\mathfrak{a}_{1}}{\mathfrak{m}}\right)\right] \\
& \leq(1-s b)^{2} \curlyvee\left(\mathfrak{a}_{2}\right) \Psi\left(\mathfrak{a}_{2}\right)+[1-(s(1-b))]^{2} \mathfrak{m}^{2} \curlyvee\left(\frac{\mathfrak{a}_{1}}{\mathfrak{m}}\right) \Psi\left(\frac{\mathfrak{a}_{1}}{\mathfrak{m}}\right) \\
& +(1-(s(1-b)))(1-(s b))\left[\mathfrak{m}^{2} \curlyvee\left(\frac{\mathfrak{a}_{1}}{\mathfrak{m}}\right) \Psi\left(\frac{\mathfrak{a}_{1}}{\mathfrak{m}}\right)+\curlyvee\left(\mathfrak{a}_{2}\right) \Psi\left(\mathfrak{a}_{2}\right)\right] \\
& =\left[(1-s b) \curlyvee\left(\mathfrak{a}_{2}\right) \Psi\left(\mathfrak{a}_{2}\right)+(1-(s(1-b))) \mathfrak{m}^{2} \Upsilon\left(\frac{\mathfrak{a}_{1}}{\mathfrak{m}}\right) \Psi\left(\frac{\mathfrak{a}_{1}}{\mathfrak{m}}\right)\right] \\
& \times((1-(s(1-b)))+(1-s b)) \\
& \leq(1-s b) \curlyvee\left(\mathfrak{a}_{2}\right) \Psi\left(\mathfrak{a}_{2}\right)+(1-(s(1-b))) \mathfrak{m}^{2} \Upsilon\left(\frac{\mathfrak{a}_{1}}{\mathfrak{m}}\right) \Psi\left(\frac{\mathfrak{a}_{1}}{\mathfrak{m}}\right) .
\end{aligned}
$$

This completes the proof.

Remark 4 Taking $\mathfrak{m}=1$ and $\mathcal{F}_{\rho, \lambda}^{\sigma}\left(\mathfrak{a}_{1}, \mathfrak{a}_{2}, \mathfrak{m}\right)=\mathfrak{a}_{1}-\mathfrak{m} \mathfrak{a}_{2}$ in Theorem 8 , then

$$
\curlyvee\left(b \mathfrak{a}_{1}+(1-b) \mathfrak{a}_{2}\right) \Psi\left(b \mathfrak{a}_{1}+(1-b) \mathfrak{a}_{2}\right) \leq[1-(s(1-b))] \curlyvee\left(\mathfrak{a}_{1}\right) \Psi\left(\mathfrak{a}_{1}\right)+[1-s b] \curlyvee\left(\mathfrak{a}_{2}\right) \Psi\left(\mathfrak{a}_{2}\right) .
$$

\section{Hermite-Hadamard type inequality via a generalized convex function of Raina type}

The principal intention of this section is to establish a novel version of Hermite-Hadamard type inequality in the mode of the newly discussed concept.

Theorem 9 Let $\Upsilon:\left[\mathfrak{a}_{1}, \mathfrak{a}_{2}\right] \in \mathbb{R}$ be a generalized s-type $\mathfrak{m}$-convex function of Raina type, if $\mathfrak{a}_{1}<\mathfrak{a}_{2}$ and $\Upsilon \in L\left[\mathfrak{a}_{1}, \mathfrak{a}_{2}\right]$, then the following Hermite-Hadamard type inequalities hold:

$$
\begin{aligned}
\curlyvee\left(\mathfrak{a}_{2}+\frac{1}{2} \mathcal{F}_{\rho, \lambda}^{\sigma}\left(\mathfrak{a}_{1}-\mathfrak{a}_{2}\right)\right) \leq & \frac{\left(1-\frac{s}{2}\right)}{\mathcal{F}_{\rho, \lambda}^{\sigma}\left(\mathfrak{a}_{1}-\mathfrak{a}_{2}\right)}\left[\int_{\mathfrak{a}_{2}}^{\mathfrak{a}_{2}+\mathcal{F}_{\rho, \lambda}^{\sigma}\left(\mathfrak{a}_{1}-\mathfrak{a}_{2}\right)} \mathfrak{m} \Upsilon\left(\frac{x}{\mathfrak{m}}\right) d x+\int_{\frac{\mathfrak{m} \mathfrak{a}_{2}+\mathcal{F}}{\mathfrak{\rho}_{, \lambda}\left(\mathfrak{a}_{1}-\mathfrak{m} \mathfrak{a}_{2}\right)} \mathfrak{\mathfrak { m }}}^{\mathfrak{a}_{2}} \curlyvee(x) d x\right] \\
& \leq(2-s)\left[\curlyvee\left(\mathfrak{a}_{2}\right)+\mathfrak{m} \Upsilon\left(\frac{\mathfrak{a}_{1}}{\mathfrak{m}}\right)\right] .
\end{aligned}
$$

Proof Since $\mathfrak{a}_{1}, \mathfrak{a}_{2} \in \mathbb{X}^{\circ}$ and $\mathbb{X}^{\circ}$ is a generalized convex set with respect to $\mathcal{F}_{\rho, \lambda}^{\sigma}$ for every $\mathfrak{m} \in(0,1]$ and $b \in[0,1]$, we have $\mathfrak{a}_{2}+b \mathcal{F}_{\rho, \lambda}^{\sigma}\left(\mathfrak{a}_{1}-\mathfrak{a}_{2}\right) \in \mathbb{X}^{\circ}$. For the first inequality, using the Definition of generalized s-type $\mathrm{m}$-convex function of Raina type, and condition $\mathrm{C}$ for $\mathcal{F}_{\rho, \lambda}^{\sigma}$ and integrating over $[0,1]$,

$$
\begin{aligned}
& \curlyvee\left(y+b \mathcal{F}_{\rho, \lambda}^{\sigma}(x-y)\right) \leq(1-(s b)) \curlyvee(y)+(1-(s(1-b))) \mathfrak{m} \curlyvee\left(\frac{x}{\mathfrak{m}}\right) \\
& \curlyvee\left(y+\frac{1}{2} \mathcal{F}_{\rho, \lambda}^{\sigma}(x-y)\right) \leq\left(1-\frac{s}{2}\right)\left[\mathfrak{m} \Upsilon\left(\frac{x}{\mathfrak{m}}\right)+\curlyvee(y)\right] .
\end{aligned}
$$

Put $x=\mathfrak{a}_{2}+b \mathcal{F}_{\rho, \lambda}^{\sigma}\left(\mathfrak{a}_{1}-\mathfrak{a}_{2}\right)$ and $\mathfrak{m} y=\mathfrak{m} \mathfrak{a}_{2}+(1-b) \mathcal{F}_{\rho, \lambda}^{\sigma}\left(\mathfrak{a}_{1}-\mathfrak{m} \mathfrak{a}_{2}\right)$ in above inequality, the L.H.S of above inequality becomes

$$
\Upsilon\left(y+\frac{1}{2} \mathcal{F}_{\rho, \lambda}^{\sigma}(x-y)\right)=\Upsilon\left(\mathfrak{a}_{2}+\frac{1}{2} \mathcal{F}_{\rho, \lambda}^{\sigma}\left(\mathfrak{a}_{1}-\mathfrak{a}_{2}\right)\right)
$$


Now,

$$
\begin{aligned}
& \curlyvee\left(\mathfrak{a}_{2}+\frac{1}{2} \mathcal{F}_{\rho, \lambda}^{\sigma}\left(\mathfrak{a}_{1}-\mathfrak{a}_{2}\right)\right) \\
& \leq\left(1-\frac{s}{2}\right)\left[\int_{0}^{1} \mathfrak{m} \Upsilon\left(\frac{\mathfrak{a}_{2}+b \mathcal{F}_{\rho, \lambda}^{\sigma}\left(\mathfrak{a}_{1}-\mathfrak{a}_{2}\right)}{\mathfrak{m}}\right) d b+\int_{0}^{1} \curlyvee\left(\mathfrak{a}_{2}+\frac{(1-b)}{\mathfrak{m}} \mathcal{F}_{\rho, \lambda}^{\sigma}\left(\mathfrak{a}_{1}-\mathfrak{m} \mathfrak{a}_{2}\right)\right) d b\right]
\end{aligned}
$$

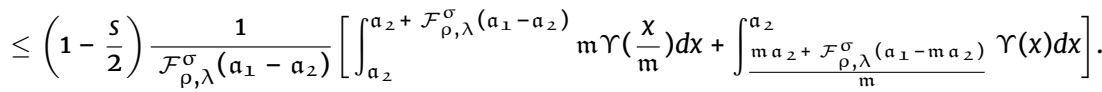

For the proof of the second inequality, using the definition of generalized $s$-type $\mathfrak{m}$ convex function, as a result we attain

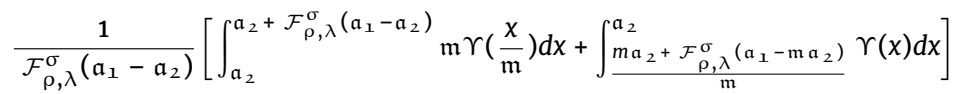

$$
\begin{aligned}
& \leq\left[\int_{0}^{1} \Upsilon\left(\mathfrak{a}_{2}+b \mathcal{F}_{\rho, \lambda}^{\sigma}\left(\mathfrak{a}_{1}-\mathfrak{a}_{2}\right)\right) d b+\int_{0}^{1} \Upsilon\left(\mathfrak{a}_{2}+\frac{(1-b)}{\mathfrak{m}} \mathcal{F}_{\rho, \lambda}^{\sigma}\left(\mathfrak{a}_{1}-\mathfrak{m} \mathfrak{a}_{2}\right)\right) d b\right] \\
& \leq \int_{0}^{1}(1-(s b)) \curlyvee\left(\mathfrak{a}_{2}\right) d b+\int_{0}^{1}(1-(s(1-b))) \mathfrak{m} \Upsilon\left(\frac{\mathfrak{a}_{1}}{\mathfrak{m}}\right) d b \\
& +\int_{0}^{1}(1-(s(1-b))) \curlyvee\left(\mathfrak{a}_{2}\right) d b+\int_{0}^{1}(1-s b) \mathfrak{m} \Upsilon\left(\frac{\mathfrak{a}_{1}}{\mathfrak{m}}\right) d b \\
& \leq\left(\frac{2-s}{2}\right)\left[\curlyvee\left(\mathfrak{a}_{2}\right)+\Upsilon\left(\mathfrak{a}_{2}\right)+\mathfrak{m} \Upsilon\left(\frac{\mathfrak{a}_{1}}{\mathfrak{m}}\right)+\mathfrak{m} \Upsilon\left(\frac{\mathfrak{a}_{1}}{\mathfrak{m}}\right)\right] \\
& \leq(2-s)\left[\curlyvee\left(\mathfrak{a}_{2}\right)+\mathfrak{m} \Upsilon\left(\frac{\mathfrak{a}_{1}}{\mathfrak{m}}\right)\right] \text {. }
\end{aligned}
$$

This completes the proof.

Corollary 1 If $s=m=1$ and $\mathcal{F}_{\rho, \lambda}^{\sigma}\left(\mathfrak{a}_{1}-\mathfrak{a}_{2}\right)=\mathfrak{a}_{1}-\mathfrak{a}_{2}$ in Theorem 9, then as a result, we attain the classical Hermite-Hadamard type inequality in [12].

\section{Estimations of Hermite-Hadamard type inequality}

The subjective aim of this section is to derive the estimations of $(\mathrm{H}-\mathrm{H})$ type inequality for a generalized $s$-type $\mathfrak{m}$-convex function of Raina type.

Lemma 2 Let $X \subseteq \mathbb{R}$ be a generalized convex subset w.r.t. $\mathcal{F}_{\rho, \lambda}^{\sigma}: X \times X \rightarrow \mathbb{R}$ and $\mathfrak{a}_{1}, \mathfrak{a}_{2} \in X$ with $\mathcal{F}_{\rho, \lambda}^{\sigma}\left(\mathfrak{a}_{1}-\mathfrak{a}_{2}\right) \neq 0$. Suppose that $\Upsilon: X \rightarrow \mathbb{R}$ is a differentiable function. If $\checkmark$ is integrable on the $\mathcal{F}_{\rho, \lambda}^{\sigma}$, then the following equality holds:

$$
\begin{aligned}
& -\frac{\Upsilon\left(\mathfrak{a}_{2}\right)+\Upsilon\left(\mathfrak{a}_{2}+\mathcal{F}_{\rho, \lambda}^{\sigma}\left(\mathfrak{a}_{1}-\mathfrak{a}_{2}\right)\right)}{2}+\frac{1}{\mathcal{F}_{\rho, \lambda}^{\sigma}\left(\mathfrak{a}_{1}-\mathfrak{a}_{2}\right)} \int_{\mathfrak{a}_{2}}^{\mathfrak{a}_{2}+\mathcal{F}_{\rho, \lambda}^{\sigma}\left(\mathfrak{a}_{1}-\mathfrak{a}_{2}\right)} \curlyvee(x) d x \\
& =\frac{\mathcal{F}_{\rho, \lambda}^{\sigma}\left(\mathfrak{a}_{1}-\mathfrak{a}_{2}\right)}{2} \int_{0}^{1}(1-2 b) \Upsilon^{\prime}\left(\mathfrak{a}_{2}+b \mathcal{F}_{\rho, \lambda}^{\sigma}\left(\mathfrak{a}_{1}-\mathfrak{a}_{2}\right)\right) d b .
\end{aligned}
$$

Proof Suppose that $\mathfrak{a}_{1}, \mathfrak{a}_{2} \in X$. Since $X$ is a generalized convex set w.r.t. $\mathcal{F}_{\rho, \lambda}^{\sigma}$, for every $b \in[0,1]$, we have $\mathfrak{a}_{2}+b \mathcal{F}_{\rho, \lambda}^{\sigma}\left(\mathfrak{a}_{1}-\mathfrak{a}_{2}\right) \in X$. Integrating by parts

$$
\begin{aligned}
& \int_{0}^{1}(1-2 b) \Upsilon^{\prime}\left(\mathfrak{a}_{2}+b \mathcal{F}_{\rho, \lambda}^{\sigma}\left(\mathfrak{a}_{1}-\mathfrak{a}_{2}\right)\right) d b \\
& =\left[\frac{(1-2 b) \Upsilon\left(\mathfrak{a}_{2}+b \mathcal{F}_{\rho, \lambda}^{\sigma}\left(\mathfrak{a}_{1}-\mathfrak{a}_{2}\right)\right)}{\mathcal{F}_{\rho, \lambda}^{\sigma}\left(\mathfrak{a}_{1}-\mathfrak{a}_{2}\right)}\right]_{0}^{1}+\frac{2}{\mathcal{F}_{\rho, \lambda}^{\sigma}\left(\mathfrak{a}_{1}-\mathfrak{a}_{2}\right)} \int_{0}^{1} \Upsilon\left(\mathfrak{a}_{2}+b \mathcal{F}_{\rho, \lambda}^{\sigma}\left(\mathfrak{a}_{1}-\mathfrak{a}_{2}\right)\right) d b \\
& =-\frac{\Upsilon\left(\mathfrak{a}_{2}\right)+\Upsilon\left(\mathfrak{a}_{2}+\mathcal{F}_{\rho, \lambda}^{\sigma}\left(\mathfrak{a}_{1}-\mathfrak{a}_{2}\right)\right)}{2}+\frac{1}{\mathcal{F}_{\rho, \lambda}^{\sigma}\left(\mathfrak{a}_{1}-\mathfrak{a}_{2}\right)} \int_{\mathfrak{a}_{2}}^{\mathfrak{a}_{2}+\mathcal{F}_{\rho, \lambda}^{\sigma}\left(\mathfrak{a}_{1}-\mathfrak{a}_{2}\right)} \Upsilon(x) d x .
\end{aligned}
$$

This completes the proof.

Theorem 10 Suppose $I^{\circ}$ is a generalized convex set w.r.t. $\mathcal{F}_{\rho, \lambda}^{\sigma}$ and $\curlyvee: I^{\circ} \subseteq \mathbb{R} \rightarrow \mathbb{R}$ is a differentiable mapping on $I^{\circ}, \mathfrak{a}_{1}, \mathfrak{a}_{2} \in I^{\circ}$ with $\mathfrak{a}_{1}<\mathfrak{a}_{2}$ and suppose that $\Upsilon^{\prime} \in L\left[\mathfrak{a}_{1}, \mathfrak{a}_{2}\right]$. If $\left|\Upsilon^{\prime}\right|$ is a generalized s-type $\mathfrak{m}$-convex function of Raina type on $L\left[\mathfrak{a}_{1}, \mathfrak{a}_{2}\right]$ for $b \in[0,1], \mathfrak{m} \in(0,1]$ and $s \in[0,1]$, then

$$
\begin{aligned}
& \left|\frac{\Upsilon\left(\mathfrak{a}_{2}\right)+\Upsilon\left(\mathfrak{a}_{2}+\mathcal{F}_{\rho, \lambda}^{\sigma}\left(\mathfrak{a}_{1}-\mathfrak{a}_{2}\right)\right)}{2}-\frac{1}{\mathcal{F}_{\rho, \lambda}^{\sigma}\left(\mathfrak{a}_{1}-\mathfrak{a}_{2}\right)} \int_{\mathfrak{a}_{2}}^{\mathfrak{a}_{2}+\mathcal{F}_{\rho, \lambda}^{\sigma}\left(\mathfrak{a}_{1}-\mathfrak{a}_{2}\right)} \curlyvee(x) d x\right| \\
& \leq\left|\mathcal{F}_{\rho, \lambda}^{\sigma}\left(\mathfrak{a}_{1}-\mathfrak{a}_{2}\right)\right|\left(\frac{2-s}{4}\right) A\left(\mathfrak{m}\left|\Upsilon^{\prime}\left(\frac{\mathfrak{a}_{1}}{\mathfrak{m}}\right)\right|,\left|\Upsilon^{\prime}\left(\mathfrak{a}_{2}\right)\right|\right),
\end{aligned}
$$

where $A$ is the arithmetic mean. 
Proof Assume that $\mathfrak{a}_{1}, \mathfrak{a}_{2} \in I^{\circ}$. Since $I^{\circ}$ is a generalized convex set w.r.t. $\mathcal{F}_{\rho, \lambda}^{\sigma}$, for any $b \in[0,1]$, we have $\mathfrak{a}_{2}+b \mathcal{F}_{\rho, \lambda}^{\sigma}\left(\mathfrak{a}_{1}-\mathfrak{a}_{2}\right) \in I^{\circ}$. Using Lemma 2, generalized s-type $m$-convex function of Raina type of $\left|\gamma^{\prime}\right|$ and properties of modulus, we have

$$
\begin{aligned}
& \left|\frac{\Upsilon\left(\mathfrak{a}_{2}\right)+\Upsilon\left(\mathfrak{a}_{2}+\mathcal{F}_{\rho, \lambda}^{\sigma}\left(\mathfrak{a}_{1}-\mathfrak{a}_{2}\right)\right)}{2}-\frac{1}{\mathcal{F}_{\rho, \lambda}^{\sigma}\left(\mathfrak{a}_{1}-\mathfrak{a}_{2}\right)} \int_{\mathfrak{a}_{2}}^{\mathfrak{a}_{2}+\mathcal{F}_{\rho, \lambda}^{\sigma}\left(\mathfrak{a}_{1}-\mathfrak{a}_{2}\right)} \Upsilon(x) d x\right| \\
& \leq\left|\frac{\mathcal{F}_{\rho, \lambda}^{\sigma}\left(\mathfrak{a}_{1}-\mathfrak{a}_{2}\right)}{2} \int_{0}^{1}(1-2 b) \Upsilon^{\prime}\left(\mathfrak{a}_{2}+b \mathcal{F}_{\rho, \lambda}^{\sigma}\left(\mathfrak{a}_{1}-\mathfrak{a}_{2}\right)\right) d b\right| \\
& \leq \frac{\left|\mathcal{F}_{\rho, \lambda}^{\sigma}\left(\mathfrak{a}_{1}-\mathfrak{a}_{2}\right)\right|}{2} \int_{0}^{1}|1-2 b|\left((1-(s b))\left|\Upsilon^{\prime}\left(\mathfrak{a}_{2}\right)\right|+(1-(s(1-b))) \mathfrak{m}\left|\Upsilon^{\prime}\left(\frac{\mathfrak{a}_{1}}{\mathfrak{m}}\right)\right|\right) d b \\
& \leq \frac{\left|\mathcal{F}_{\rho, \lambda}^{\sigma}\left(\mathfrak{a}_{1}-\mathfrak{a}_{2}\right)\right|}{2}\left(\left|\Upsilon^{\prime}\left(\mathfrak{a}_{2}\right)\right| \int_{0}^{1}|1-2 b|(1-(s b)) d b+\mathfrak{m}\left|\Upsilon^{\prime}\left(\frac{\mathfrak{a}_{1}}{\mathfrak{m}}\right)\right| \int_{0}^{1}|1-2 b|(1-(s(1-b))) d b\right) \\
& \leq \frac{\left|\mathcal{F}_{\rho, \lambda}^{\sigma}\left(\mathfrak{a}_{1}-\mathfrak{a}_{2}\right)\right|}{2}\left(\left|\Upsilon^{\prime}\left(\mathfrak{a}_{2}\right)\right|\left(\frac{2-s}{4}\right)+\left|\mathfrak{m} \Upsilon^{\prime}\left(\frac{\mathfrak{a}_{1}}{\mathfrak{m}}\right)\right|\left(\frac{2-s}{4}\right)\right) \\
& \leq\left|\mathcal{F}_{\rho, \lambda}^{\sigma}\left(\mathfrak{a}_{1}-\mathfrak{a}_{2}\right)\right|\left(\frac{2-s}{4}\right) A\left(\mathfrak{m}\left|\Upsilon^{\prime}\left(\frac{\mathfrak{a}_{1}}{\mathfrak{m}}\right)\right|,\left|\Upsilon^{\prime}\left(\mathfrak{a}_{2}\right)\right|\right) .
\end{aligned}
$$

This is the required proof.

Corollary 2 If we put $\mathfrak{m}=1$ and $s=1$ in Theorem 10 , then we obtain Theorem (2.1) in [17].

Corollary 3 If we put $s=\mathfrak{m}=1$ and $\mathcal{F}_{\rho, \lambda}^{\sigma}\left(\mathfrak{a}_{1}-\mathfrak{a}_{2}\right)=\mathfrak{a}_{1}-\mathfrak{a}_{2}$ in Theorem 10, we get inequality (4.1) in [18].

Theorem 11 Suppose $I^{\circ}$ is a generalized convex set w.r.t. $\mathcal{F}_{\rho, \lambda}^{\sigma}$ and $\Upsilon: I^{\circ} \subseteq \mathbb{R} \rightarrow \mathbb{R}$ be a differentiable mapping on $I^{\circ}, \mathfrak{a}_{1}, \mathfrak{a}_{2} \in I^{\circ}$ with $\mathfrak{a}_{1}<\mathfrak{a}_{2}, q>1, \frac{1}{p}+\frac{1}{q}=1$ and suppose that $\gamma^{\prime} \in L\left[\mathfrak{a}_{1}, \mathfrak{a}_{2}\right]$. If $\left|\gamma^{\prime}\right|^{q}$ is a generalized s-type $\mathfrak{m}$-convex function of Raina type on $L\left[\mathfrak{a}_{1}, \mathfrak{a}_{2}\right]$ for $b \in[0,1], \mathfrak{m} \in(0,1]$ and $s \in[0,1]$, then

$$
\begin{aligned}
& \left|\frac{\Upsilon\left(\mathfrak{a}_{2}\right)+\Upsilon\left(\mathfrak{a}_{2}+\mathcal{F}_{\rho, \lambda}^{\sigma}\left(\mathfrak{a}_{1}-\mathfrak{a}_{2}\right)\right)}{2}-\frac{1}{\mathcal{F}_{\rho, \lambda}^{\sigma}\left(\mathfrak{a}_{1}-\mathfrak{a}_{2}\right)} \int_{\mathfrak{a}_{2}}^{\mathfrak{a}_{2}+\mathcal{F}_{\rho, \lambda}^{\sigma}\left(\mathfrak{a}_{1}-\mathfrak{a}_{2}\right)} \curlyvee(x) d x\right| \\
& \leq \frac{\left|\mathcal{F}_{\rho, \lambda}^{\sigma}\left(\mathfrak{a}_{1}-\mathfrak{a}_{2}\right)\right|}{2}\left(\frac{1}{p+1}\right)^{\frac{1}{p}}\left(\frac{2-s}{2}\right)^{\frac{1}{q}} A^{\frac{1}{q}}\left(\mathfrak{m}\left|\gamma^{\prime}\left(\frac{\mathfrak{a}_{1}}{\mathfrak{m}}\right)\right|^{q},\left|\Upsilon^{\prime}\left(\mathfrak{a}_{2}\right)\right|^{q}\right),
\end{aligned}
$$

where $A$ is the arithmetic mean.

Proof Assume that $\mathfrak{a}_{1}, \mathfrak{a}_{2} \in I^{\circ}$. Since $I^{\circ}$ is a generalized convex set w.r.t. $\mathcal{F}_{\rho, \lambda}^{\sigma}$, for any $b \in[0,1]$, we have $\mathfrak{a}_{2}+b \mathcal{F}_{\rho, \lambda}^{\sigma}\left(\mathfrak{a}_{1}-\mathfrak{a}_{2}\right) \in I^{\circ}$. Using Lemma 2, Hölder's integral inequality and generalized s-type $\mathfrak{m}$-convex function of Raina type of $\left|\Upsilon^{\prime}\right|^{q}$ and properties of modulus, we have

$$
\begin{aligned}
& \left|\frac{\Upsilon\left(\mathfrak{a}_{2}\right)+\Upsilon\left(\mathfrak{a}_{2}+\mathcal{F}_{\rho, \lambda}^{\sigma}\left(\mathfrak{a}_{1}-\mathfrak{a}_{2}\right)\right)}{2}-\frac{1}{\mathcal{F}_{\rho, \lambda}^{\sigma}\left(\mathfrak{a}_{1}-\mathfrak{a}_{2}\right)} \int_{\mathfrak{a}_{2}}^{\mathfrak{a}_{2}+\mathcal{F}_{\rho, \lambda}^{\sigma}\left(\mathfrak{a}_{1}-\mathfrak{a}_{2}\right)} \curlyvee(x) d x\right| \\
& \leq\left|\frac{\mathcal{F}_{\rho, \lambda}^{\sigma}\left(\mathfrak{a}_{1}-\mathfrak{a}_{2}\right)}{2} \int_{0}^{1}(1-2 b) \Upsilon^{\prime}\left(\mathfrak{a}_{2}+b \mathcal{F}_{\rho, \lambda}^{\sigma}\left(\mathfrak{a}_{1}-\mathfrak{a}_{2}\right)\right) d b\right| \\
& \leq \frac{\left|\mathcal{F}_{\rho, \lambda}^{\sigma}\left(\mathfrak{a}_{1}-\mathfrak{a}_{2}\right)\right|}{2}\left(\int_{0}^{1}|1-2 b|^{p}\right)^{\frac{1}{p}}\left(\int_{0}^{1}\left|\Upsilon^{\prime}\left(\mathfrak{a}_{2}+b \mathcal{F}_{\rho, \lambda}^{\sigma}\left(\mathfrak{a}_{1}-\mathfrak{a}_{2}\right)\right)\right|^{q} d b\right)^{\frac{1}{q}} \\
& \leq \frac{\left|\mathcal{F}_{\rho, \lambda}^{\sigma}\left(\mathfrak{a}_{1}-\mathfrak{a}_{2}\right)\right|}{2}\left(\frac{1}{p+1}\right)^{\frac{1}{p}}\left(\left|\Upsilon^{\prime}\left(\mathfrak{a}_{2}\right)\right|^{q} \int_{0}^{1}(1-(s b)) d b+\int_{0}^{1} \mathfrak{m}\left|\Upsilon^{\prime}\left(\frac{\mathfrak{a}_{1}}{\mathfrak{m}}\right)\right|^{q}(1-(s(1-b))) d b\right)^{\frac{1}{q}} \\
& \leq \frac{\left|\mathcal{F}_{\rho, \lambda}^{\sigma}\left(\mathfrak{a}_{1}-\mathfrak{a}_{2}\right)\right|}{2}\left(\frac{1}{p+1}\right)^{\frac{1}{p}}\left(\frac{2-s}{2}\right)^{\frac{1}{q}} A^{\frac{1}{q}}\left(\mathfrak{m}\left|\Upsilon^{\prime}\left(\frac{\mathfrak{a}_{1}}{\mathfrak{m}}\right)\right|^{q},\left|\Upsilon^{\prime}\left(\mathfrak{a}_{2}\right)\right|^{q}\right),
\end{aligned}
$$

which is the required proof.

Corollary 4 If $\mathfrak{m}=1$ and $s=1$ in Theorem 11, then we attain Theorem (2.2) in [17]

Corollary 5 If we put $\mathfrak{m}=s=1$ and $\mathcal{F}_{\rho, \lambda}^{\sigma}\left(\mathfrak{a}_{1}-\mathfrak{a}_{2}\right)=\mathfrak{a}_{1}-\mathfrak{a}_{2}$ in Theorem 11, then we get inequality (4.2) [18]

Theorem 12 Suppose $I^{\circ}$ is a generalized convex set w.r.t. $\quad \mathcal{F}_{\rho, \lambda}^{\sigma}$ and $\curlyvee: I^{\circ} \subseteq \mathbb{R} \rightarrow \mathbb{R}$ is a differentiable mapping on $I^{\circ}, \mathfrak{a}_{1}, \mathfrak{a}_{2} \in I^{\circ}$ with $\mathfrak{a}_{1}<\mathfrak{a}_{2}, q \geq 1$, and suppose that $\Upsilon^{\prime} \in L\left[\mathfrak{a}_{1}, \mathfrak{a}_{2}\right]$. If $\left|\Upsilon^{\prime}\right|^{q}$ is a generalized s-type $\mathfrak{m}$-convex function of Raina type on $L\left[\mathfrak{a}_{1}, \mathfrak{a}_{2}\right]$ for for $b \in[0,1]$, $\mathfrak{m} \in(0,1]$ and $s \in[0,1]$, then

$$
\begin{aligned}
& \left|\frac{\Upsilon\left(\mathfrak{a}_{2}\right)+\Upsilon\left(\mathfrak{a}_{2}+\mathcal{F}_{\rho, \lambda}^{\sigma}\left(\mathfrak{a}_{1}-\mathfrak{a}_{2}\right)\right)}{2}-\frac{1}{\mathcal{F}_{\rho, \lambda}^{\sigma}\left(\mathfrak{a}_{1}-\mathfrak{a}_{2}\right)} \int_{\mathfrak{a}_{2}}^{\mathfrak{a}_{2}+\mathcal{F}_{\rho, \lambda}^{\sigma}\left(\mathfrak{a}_{1}-\mathfrak{a}_{2}\right)} \curlyvee(x) d x\right| \\
& \leq \frac{\left|\mathcal{F}_{\rho, \lambda}^{\sigma}\left(\mathfrak{a}_{1}-\mathfrak{a}_{2}\right)\right|}{2}\left(\frac{1}{2}\right)^{1-\frac{2}{q}}\left(\frac{2-s}{4}\right)^{\frac{1}{q}} A^{\frac{1}{q}}\left(\mathfrak{m}\left|\gamma^{\prime}\left(\frac{\mathfrak{a}_{1}}{\mathfrak{m}}\right)\right|^{q},\left|\Upsilon^{\prime}\left(\mathfrak{a}_{2}\right)\right|^{q}\right),
\end{aligned}
$$

where $A$ is the arithmetic mean. 
Proof Assume that $\mathfrak{a}_{1}, \mathfrak{a}_{2} \in I^{\circ}$. Since $I^{\circ}$ is a generalized convex set w.r.t. $\mathcal{F}_{\rho, \lambda}^{\sigma}$, for any $b \in[0,1]$, we have $\mathfrak{a}_{2}+b \mathcal{F}_{\rho, \lambda}^{\sigma}\left(\mathfrak{a}_{1}-\mathfrak{a}_{2}\right) \in I^{\circ}$. Suppose that $q>1$. Using Lemma 2, power-mean inequality and generalized $s$-type $\mathfrak{m}$-convex function of Raina type of $\left|\gamma^{\prime}\right|^{q}$ and properties of modulus, we have

$$
\begin{aligned}
& \left|\frac{\Upsilon\left(\mathfrak{a}_{2}\right)+\Upsilon\left(\mathfrak{a}_{2}+\mathcal{F}_{\rho, \lambda}^{\sigma}\left(\mathfrak{a}_{1}-\mathfrak{a}_{2}\right)\right)}{2}-\frac{1}{\mathcal{F}_{\rho, \lambda}^{\sigma}\left(\mathfrak{a}_{1}-\mathfrak{a}_{2}\right)} \int_{\mathfrak{a}_{2}}^{\mathfrak{a}_{2}+\mathcal{F}_{\rho, \lambda}^{\sigma}\left(\mathfrak{a}_{1}-\mathfrak{a}_{2}\right)} \curlyvee(x) d x\right| \\
& \leq\left|\frac{\mathcal{F}_{\rho, \lambda}^{\sigma}\left(\mathfrak{a}_{1}-\mathfrak{a}_{2}\right)}{2} \int_{0}^{1}(1-2 b) \Upsilon^{\prime}\left(\mathfrak{a}_{2}+b \mathcal{F}_{\rho, \lambda}^{\sigma}\left(\mathfrak{a}_{1}-\mathfrak{a}_{2}\right)\right) d b\right| \\
& \leq \frac{\left|\mathcal{F}_{\rho, \lambda}^{\sigma}\left(\mathfrak{a}_{1}-\mathfrak{a}_{2}\right)\right|}{2}\left(\int_{0}^{1}|1-2 b| d b\right)^{1-\frac{1}{q}}\left(\int_{0}^{1}|1-2 b|\left|\Upsilon^{\prime}\left(\mathfrak{a}_{2}+b \mathcal{F}_{\rho, \lambda}^{\sigma}\left(\mathfrak{a}_{1}-\mathfrak{a}_{2}\right)\right)\right|^{q} d b\right)^{\frac{1}{q}} \\
& \leq \frac{\left|\mathcal{F}_{\rho, \lambda}^{\sigma}\left(\mathfrak{a}_{1}-\mathfrak{a}_{2}\right)\right|}{2}\left(\frac{1}{2}\right)^{1-\frac{1}{q}}\left(\int_{0}^{1}|1-2 b|\left[(1-(s b))\left|\Upsilon^{\prime}\left(\mathfrak{a}_{2}\right)\right|^{q}+(1-(s(1-b)))\left|\Upsilon^{\prime}\left(\mathfrak{a}_{1}\right)\right|^{q}\right] d b\right)^{\frac{1}{q}} \\
& \leq \frac{\left|\mathcal{F}_{\rho, \lambda}^{\sigma}\left(\mathfrak{a}_{1}-\mathfrak{a}_{2}\right)\right|}{2}\left(\frac{1}{2}\right)^{1-\frac{1}{q}} \\
& \times\left(\left|\mathcal{\gamma}^{\prime}\left(\mathfrak{a}_{2}\right)\right|^{q} \int_{0}^{1}|1-2 b|(1-(s b)) d b+\int_{0}^{1}|1-2 b| \mathfrak{m}\left|\Upsilon^{\prime}\left(\frac{\mathfrak{a}_{1}}{\mathfrak{m}}\right)\right|^{q}(1-(s(1-b))) d b\right)^{\frac{1}{q}} \\
& =\frac{\left|\mathcal{F}_{\rho, \lambda}^{\sigma}\left(\mathfrak{a}_{1}-\mathfrak{a}_{2}\right)\right|}{2}\left(\frac{1}{2}\right)^{1-\frac{2}{q}}\left(\frac{2-s}{4}\right)^{\frac{1}{q}} A^{\frac{1}{q}}\left(\mathfrak{m}\left|\Upsilon^{\prime}\left(\frac{\mathfrak{a}_{1}}{\mathfrak{m}}\right)\right|^{q},\left|\Upsilon^{\prime}\left(\mathfrak{a}_{2}\right)\right|^{q}\right) .
\end{aligned}
$$

For the case $q=1$, we apply the same technique step by step as used in the Theorem 10 . This completes the proof.

Corollary 6 If we put $n=m=1$ and $s=1$ in Theorem 12 , then

$$
\begin{aligned}
& \left|\frac{\Upsilon\left(\mathfrak{a}_{2}\right)+\Upsilon\left(\mathfrak{a}_{2}+\mathcal{F}_{\rho, \lambda}^{\sigma}\left(\mathfrak{a}_{1}-\mathfrak{a}_{2}\right)\right)}{2}-\frac{1}{\mathcal{F}_{\rho, \lambda}^{\sigma}\left(\mathfrak{a}_{1}-\mathfrak{a}_{2}\right)} \int_{\mathfrak{a}_{2}}^{\mathfrak{a}_{2}+\mathcal{F}_{\rho, \lambda}^{\sigma}\left(\mathfrak{a}_{1}-\mathfrak{a}_{2}\right)} \curlyvee(x) d x\right| \\
& \leq \frac{\mathcal{F}_{\rho, \lambda}^{\sigma}\left(\mathfrak{a}_{1}-\mathfrak{a}_{2}\right)}{4} A^{\frac{1}{q}}\left[\left|\Upsilon^{\prime}\left(\mathfrak{a}_{1}\right)\right|^{q},\left|\Upsilon^{\prime}\left(\mathfrak{a}_{2}\right)\right|^{q}\right] .
\end{aligned}
$$

Corollary 7 If $s=\mathfrak{m}=1$ and $\mathcal{F}_{\rho, \lambda}^{\sigma}\left(\mathfrak{a}_{1}-\mathfrak{a}_{2}\right)=\mathfrak{a}_{1}-\mathfrak{a}_{2}$ in Theorem 12, we get inequality (4.3) in [18].

Theorem 13 Suppose $I^{\circ}$ is a generalized convex set w.r.t. $\quad \mathcal{F}_{\rho, \lambda}^{\sigma}$ and $\gamma: I^{\circ} \subseteq \mathbb{R} \rightarrow \mathbb{R}$ is a differentiable mapping on $I^{\circ}, \mathfrak{a}_{1}, \mathfrak{a}_{2} \in I^{\circ}$ with $\mathfrak{a}_{1}<\mathfrak{a}_{2}, q>1, \frac{1}{p}+\frac{1}{q}=1$ and suppose that $\Upsilon^{\prime} \in L\left[\mathfrak{a}_{1}, \mathfrak{a}_{2}\right]$. If $\left|\Upsilon^{\prime}\right| q$ is a generalized $s$-type $\mathfrak{m}$-convex function of Raina type on $L\left[\mathfrak{a}_{1}, \mathfrak{a}_{2}\right]$, then

$$
\begin{aligned}
& \left|\frac{\Upsilon\left(\mathfrak{a}_{2}\right)+\Upsilon\left(\mathfrak{a}_{2}+\mathcal{F}_{\rho, \lambda}^{\sigma}\left(\mathfrak{a}_{1}-\mathfrak{a}_{2}\right)\right)}{2}-\frac{1}{\mathcal{F}_{\rho, \lambda}^{\sigma}\left(\mathfrak{a}_{1}-\mathfrak{a}_{2}\right)} \int_{\mathfrak{a}_{2}}^{\mathfrak{a}_{2}+\mathcal{F}_{\rho, \lambda}^{\sigma}\left(\mathfrak{a}_{1}-\mathfrak{a}_{2}\right)} \curlyvee(x) d x\right| \\
& \leq \frac{\left|\mathcal{F}_{\rho, \lambda}^{\sigma}\left(\mathfrak{a}_{1}-\mathfrak{a}_{2}\right)\right|}{2}\left(\frac{1}{2(p+1)}\right)^{\frac{1}{p}} \\
& \times\left\{\left(\mathfrak{m}\left|\Upsilon^{\prime}\left(\frac{\mathfrak{a}_{1}}{\mathfrak{m}}\right)\right|^{q} \frac{3-2 s}{6}+\left|\Upsilon^{\prime}\left(\mathfrak{a}_{2}\right)\right|^{q} \frac{3-s}{6}\right)^{\frac{1}{q}}+\left(\mathfrak{m}\left|\Upsilon^{\prime}\left(\frac{\mathfrak{a}_{1}}{\mathfrak{m}}\right)\right|^{q} \frac{3-s}{6}+\left|\Upsilon^{\prime}\left(\mathfrak{a}_{2}\right)\right|^{q} \frac{3-2 s}{6}\right)^{\frac{1}{q}}\right\},
\end{aligned}
$$

holds for $b \in[0,1], \mathfrak{m} \in(0,1]$ and $s \in[0,1]$.

Proof Suppose that $\mathfrak{a}_{1}, \mathfrak{a}_{2} \in I^{\circ}$. Since $I^{\circ}$ is a generalized convex set w.r.t. $\mathcal{F}_{\rho, \lambda}^{\sigma}$, for any $b \in[0,1]$, we have $\mathfrak{a}_{2}+b \mathcal{F}_{\rho, \lambda}^{\sigma}\left(\mathfrak{a}_{1}-\mathfrak{a}_{2}\right) \in I^{\circ}$. Using Lemma 2, Hölder-İscan integral inequality and generalized s-type $\mathfrak{m}$-convex function of Raina type of $\left|\gamma^{\prime}\right| q$ and properties 
of modulus, we have

$$
\begin{aligned}
& \left|\frac{\Upsilon\left(\mathfrak{a}_{2}\right)+\Upsilon\left(\mathfrak{a}_{2}+\mathcal{F}_{\rho, \lambda}^{\sigma}\left(\mathfrak{a}_{1}-\mathfrak{a}_{2}\right)\right)}{2}-\frac{1}{\mathcal{F}_{\rho, \lambda}^{\sigma}\left(\mathfrak{a}_{1}-\mathfrak{a}_{2}\right)} \int_{\mathfrak{a}_{2}}^{\mathfrak{a}_{2}+\mathcal{F}_{\rho, \lambda}^{\sigma}\left(\mathfrak{a}_{1}-\mathfrak{a}_{2}\right)} \Upsilon(x) d x\right| \\
& \leq \frac{\left|\mathcal{F}_{\rho, \lambda}^{\sigma}\left(\mathfrak{a}_{1}-\mathfrak{a}_{2}\right)\right|}{2} \int_{0}^{1}|1-2 b|\left|\gamma^{\prime}\left(\mathfrak{a}_{2}+b \mathcal{F}_{\rho, \lambda}^{\sigma}\left(\mathfrak{a}_{1}-\mathfrak{a}_{2}\right)\right)\right| d b \\
& \leq \frac{\left|\mathcal{F}_{\rho, \lambda}^{\sigma}\left(a_{1}-\mathfrak{a}_{2}\right)\right|}{2}\left(\int_{0}^{1}(1-b)|1-2 b|^{p} d b\right)^{\frac{1}{p}}\left(\int_{0}^{1}(1-b)\left|\gamma^{\prime}\left(a_{2}+b \mathcal{F}_{\rho, \lambda}^{\sigma}\left(\mathfrak{a}_{1}-\mathfrak{a}_{2}\right)\right)\right|^{q} d b\right)^{\frac{1}{q}} \\
& +\frac{\left|\mathcal{F}_{\rho, \lambda}^{\sigma}\left(\mathfrak{a}_{1}-\mathfrak{a}_{2}\right)\right|}{2}\left(\int_{0}^{1} b|1-2 b|^{p} d b\right)^{\frac{1}{p}}\left(\int_{0}^{1} b\left|\gamma^{\prime}\left(\mathfrak{a}_{2}+b \mathcal{F}_{\rho, \lambda}^{\sigma}\left(\mathfrak{a}_{1}-\mathfrak{a}_{2}\right)\right)\right|^{q} d b\right)^{\frac{1}{q}} \\
& \leq \frac{\left|\mathcal{F}_{\rho, \lambda}^{\sigma}\left(\mathfrak{a}_{1}-\mathfrak{a}_{2}\right)\right|}{2}\left(\frac{1}{2(p+1)}\right)^{\frac{1}{p}} \\
& \times\left(\left|\Upsilon^{\prime}\left(\mathfrak{a}_{2}\right)\right|^{q} \int_{0}^{1}(1-b)(1-(s b)) d b+\int_{0}^{1}(1-b) \mathfrak{m}\left|\gamma^{\prime}\left(\frac{\mathfrak{a}_{1}}{\mathfrak{m}}\right)\right|^{q}(1-(s(1-b))) d b\right)^{\frac{1}{q}} \\
& +\frac{\left|\mathcal{F}_{\rho, \lambda}^{\sigma}\left(\mathfrak{a}_{1}-\mathfrak{a}_{2}\right)\right|}{2}\left(\frac{1}{2(p+1)}\right)^{\frac{1}{p}}\left(\left|\gamma^{\prime}\left(\mathfrak{a}_{2}\right)\right|^{q} \int_{0}^{1} b(1-(s b)) d b+\int_{0}^{1} \mathfrak{m}\left|\gamma^{\prime}\left(\frac{\mathfrak{a}_{1}}{\mathfrak{m}}\right)\right|^{q_{b}} b(1-(s(1-b))) d b\right)^{\frac{1}{q}} \\
& \leq \frac{\left|\mathcal{F}_{\rho, \lambda}^{\sigma}\left(\mathfrak{a}_{1}-\mathfrak{a}_{2}\right)\right|}{2}\left(\frac{1}{2(p+1)}\right)^{\frac{1}{p}} \\
& \times\left\{\left(\mathfrak{m}\left|\gamma^{\prime}\left(\frac{\mathfrak{a}_{1}}{\mathfrak{m}}\right)\right|^{q} \frac{3-2 s}{6}+\left|\Upsilon^{\prime}\left(\mathfrak{a}_{2}\right)\right|^{q} \frac{3-s}{6}\right)^{\frac{1}{q}}+\left(\mathfrak{m}\left|\gamma^{\prime}\left(\frac{\mathfrak{a}_{1}}{\mathfrak{m}}\right)\right|^{q} \frac{3-s}{6}+\left|\gamma^{\prime}\left(\mathfrak{a}_{2}\right)\right|^{q} \frac{3-2 s}{6}\right)^{\frac{1}{q}}\right\},
\end{aligned}
$$

which is the required proof.

Corollary 8 If $\mathfrak{m}=1$ and $s=1$ in Theorem 13 , then

$$
\begin{aligned}
& \left|\frac{\Upsilon\left(\mathfrak{a}_{2}\right)+\Upsilon\left(\mathfrak{a}_{2}+\mathcal{F}_{\rho, \lambda}^{\sigma}\left(\mathfrak{a}_{1}-\mathfrak{a}_{2}\right)\right)}{2}-\frac{1}{\mathcal{F}_{\rho, \lambda}^{\sigma}\left(\mathfrak{a}_{1}-\mathfrak{a}_{2}\right)} \int_{\mathfrak{a}_{2}}^{\mathfrak{a}_{2}+\mathcal{F}_{\rho, \lambda}^{\sigma}\left(\mathfrak{a}_{1}-\mathfrak{a}_{2}\right)} \Upsilon(x) d x\right| \\
& \leq \frac{\left|\mathcal{F}_{\rho, \lambda}^{\sigma}\left(\mathfrak{a}_{1}-\mathfrak{a}_{2}\right)\right|}{4}\left(\frac{1}{p+1}\right)^{\frac{1}{p}}\left[\left(\frac{\left|\Upsilon^{\prime}\left(\mathfrak{a}_{1}\right)\right|^{q}}{3}+\frac{2\left|\Upsilon^{\prime}\left(\mathfrak{a}_{2}\right)\right|^{q}}{3}\right)^{\frac{1}{q}}+\left(\frac{2\left|\Upsilon^{\prime}\left(\mathfrak{a}_{1}\right)\right|^{q}}{3}+\frac{\left|\Upsilon^{\prime}\left(\mathfrak{a}_{2}\right)\right|^{q}}{3}\right)^{\frac{1}{q}}\right] .
\end{aligned}
$$

Corollary 9 If we put $s=\mathfrak{m}=1$ and $\mathcal{F}_{\rho, \lambda}^{\sigma}\left(\mathfrak{a}_{1}-\mathfrak{a}_{2}\right)=\mathfrak{a}_{1}-\mathfrak{a}_{2}$ in Theorem 13, we get inequality (4.4) in [18].

Theorem 14 Suppose $I^{\circ}$ is a generalized convex set w.r.t. $\quad \mathcal{F}_{\rho, \lambda}^{\sigma}$ and $\curlyvee: I^{\circ} \subseteq \mathbb{R} \rightarrow \mathbb{R}$ is a differentiable mapping on $I^{\circ}, \mathfrak{a}_{1}, \mathfrak{a}_{2} \in I^{\circ}$ with $\mathfrak{a}_{1}<\mathfrak{a}_{2}, q \geq 1$ and suppose that $\Upsilon^{\prime} \in L\left[\mathfrak{a}_{1}, \mathfrak{a}_{2}\right]$. If $\left|\gamma^{\prime}\right|^{q}$ is a generalized s-type $\mathfrak{m}$-convex function of Raina type on $L\left[\mathfrak{a}_{1}, \mathfrak{a}_{2}\right]$, then

$$
\begin{aligned}
& \left|\frac{\Upsilon\left(\mathfrak{a}_{2}\right)+\Upsilon\left(\mathfrak{a}_{2}+\mathcal{F}_{\rho, \lambda}^{\sigma}\left(\mathfrak{a}_{1}-\mathfrak{a}_{2}\right)\right)}{2}-\frac{1}{\mathcal{F}_{\rho, \lambda}^{\sigma}\left(\mathfrak{a}_{1}-\mathfrak{a}_{2}\right)} \int_{\mathfrak{a}_{2}}^{\mathfrak{a}_{2}+\mathcal{F}_{\rho, \lambda}^{\sigma}\left(\mathfrak{a}_{1}-\mathfrak{a}_{2}\right)} \curlyvee(x) d x\right| \\
& \leq \frac{\left|\mathcal{F}_{\rho, \lambda}^{\sigma}\left(\mathfrak{a}_{1}-\mathfrak{a}_{2}\right)\right|}{2}\left(\frac{1}{2}\right)^{2-\frac{2}{q}} \\
& \times\left\{\left(\mathfrak{m}\left|\Upsilon^{\prime}\left(\frac{\mathfrak{a}_{1}}{\mathfrak{m}}\right)\right|^{q} k_{1}(s)+\left|\Upsilon^{\prime}\left(\mathfrak{a}_{2}\right)\right|^{q} k_{2}(s)\right)^{\frac{1}{q}}+\left(\mathfrak{m}\left|\Upsilon^{\prime}\left(\frac{\mathfrak{a}_{1}}{\mathfrak{m}}\right)\right|{ }^{q} k_{2}(s)+\left|\Upsilon^{\prime}\left(\mathfrak{a}_{2}\right)\right|^{q} k_{1}(s)\right)^{\frac{1}{q}}\right\},
\end{aligned}
$$

holds for $b \in[0,1], \mathfrak{m} \in(0,1]$ and $s \in[0,1]$. Where

$$
\begin{aligned}
& \left.k_{1}(s)=\int_{0}^{1}(1-b)|1-2 b|(1-(s(1-b))) d b=\int_{0}^{1} b|1-2 b|(1-(s b))\right) d b, \\
& k_{2}(s)=\int_{0}^{1} b|1-2 b|(1-(s(1-b))) d b=\int_{0}^{1}(1-b)|1-2 b|(1-(s b)) d b .
\end{aligned}
$$

Proof Suppose that $\mathfrak{a}_{1}, \mathfrak{a}_{2} \in I^{\circ}$. Since $I^{\circ}$ is a generalized convex set w.r.t. $\mathcal{F}_{\rho, \lambda}^{\sigma}$, for any $b \in[0,1]$, we have $\mathfrak{a}_{2}+b \mathcal{F}_{\rho, \lambda}^{\sigma}\left(\mathfrak{a}_{1}-\mathfrak{a}_{2}\right) \in I^{\circ}$. Suppose that $q>1$. Using Lemma 2, improved power-mean integral inequality and generalized $s$-type $m$-convex function of Raina 
type of $\left|\gamma^{\prime}\right|^{q}$ and properties of modulus, we have

$$
\begin{aligned}
& \left|\frac{\Upsilon\left(\mathfrak{a}_{2}\right)+\Upsilon\left(\mathfrak{a}_{2}+\mathcal{F}_{\rho, \lambda}^{\sigma}\left(\mathfrak{a}_{1}-\mathfrak{a}_{2}\right)\right)}{2}-\frac{1}{\mathcal{F}_{\rho, \lambda}^{\sigma}\left(\mathfrak{a}_{1}-\mathfrak{a}_{2}\right)} \int_{\mathfrak{a}_{2}}^{\mathfrak{a}_{2}+\mathcal{F}_{\rho, \lambda}^{\sigma}\left(\mathfrak{a}_{1}-\mathfrak{a}_{2}\right)} \curlyvee(x) d x\right| \\
& \leq \frac{\left|\mathcal{F}_{\rho, \lambda}^{\sigma}\left(\mathfrak{a}_{1}-\mathfrak{a}_{2}\right)\right|}{2} \int_{0}^{1}|1-2 b|\left|\Upsilon^{\prime}\left(\mathfrak{a}_{2}+b \mathcal{F}_{\rho, \lambda}^{\sigma}\left(\mathfrak{a}_{1}-\mathfrak{a}_{2}\right)\right)\right| d b \\
& \leq \frac{\left|\mathcal{F}_{\rho, \lambda}^{\sigma}\left(\mathfrak{a}_{1}-\mathfrak{a}_{2}\right)\right|}{2}\left(\int_{0}^{1}(1-b)|1-2 b| d b\right)^{1-\frac{1}{q}}\left(\int_{0}^{1}(1-b)|1-2 b|\left|\gamma^{\prime}\left(\mathfrak{a}_{2}+b \mathcal{F}_{\rho, \lambda}^{\sigma}\left(\mathfrak{a}_{1}-\mathfrak{a}_{2}\right)\right)\right|^{q} d b\right)^{\frac{1}{q}} \\
& +\frac{\left|\mathcal{F}_{\rho, \lambda}^{\sigma}\left(\mathfrak{a}_{1}-\mathfrak{a}_{2}\right)\right|}{2}\left(\int_{0}^{1}(b|1-2 b| d b)^{1-\frac{1}{q}}\left(\int_{0}^{1} b|1-2 b|\left|\gamma^{\prime}\left(\mathfrak{a}_{2}+b \mathcal{F}_{\rho, \lambda}^{\sigma}\left(\mathfrak{a}_{1}-\mathfrak{a}_{2}\right)\right)\right|^{q} d b\right)^{\frac{1}{q}}\right. \\
& \leq \frac{\left|\mathcal{F}_{\rho, \lambda}^{\sigma}\left(\mathfrak{a}_{1}-\mathfrak{a}_{2}\right)\right|}{2}\left(\frac{1}{2}\right)^{2-\frac{2}{q}} \\
& \times\left\{\left(\left|\gamma^{\prime}\left(\mathfrak{a}_{2}\right)\right|^{q} \int_{0}^{1}(1-b)|1-2 b|(1-(s b)) d b+\int_{0}^{1}(1-b)|1-2 b| \mathfrak{m}\left|\gamma^{\prime}\left(\frac{\mathfrak{a}_{1}}{\mathfrak{m}}\right)\right|^{q}(1-(s(1-b))) d b\right)^{\frac{1}{q}}\right. \\
& \left.\left.+\left(\left|\Upsilon^{\prime}\left(\mathfrak{a}_{2}\right)\right|^{q} \int_{0}^{1} b|1-2 b|(1-(s b))\right) d b+\int_{0}^{1} b|1-2 b| \mathfrak{m}\left|\Upsilon^{\prime}\left(\frac{\mathfrak{a}_{1}}{\mathfrak{m}}\right)\right|^{q}(1-(s(1-b))) d b\right)^{\frac{1}{q}}\right\} \\
& \leq \frac{\left|\mathcal{F}_{\rho, \lambda}^{\sigma}\left(\mathfrak{a}_{1}-\mathfrak{a}_{2}\right)\right|}{2}\left(\frac{1}{2}\right)^{2-\frac{2}{q}} \\
& \times\left\{\left(\mathfrak{m}\left|\Upsilon^{\prime}\left(\frac{\mathfrak{a}_{1}}{\mathfrak{m}}\right)\right|^{q^{q}} k_{1}(s)+\left|\Upsilon^{\prime}\left(\mathfrak{a}_{2}\right)\right|^{q} k_{2}(s)\right)^{\frac{1}{q}}+\left(\mathfrak{m}\left|\Upsilon^{\prime}\left(\frac{\mathfrak{a}_{1}}{\mathfrak{m}}\right)\right|^{q} k_{2}(s)+\left|\Upsilon^{\prime}\left(\mathfrak{a}_{2}\right)\right|^{q} k_{1}(s)\right)^{\frac{1}{q}}\right\} .
\end{aligned}
$$

For the case $q=1$, we apply the same technique step by step as used in the Theorem 10 . This completes the proof.

Corollary 10 If we put $\mathfrak{m}=1$ and $s=1$ in Theorem 14 , then

$$
\begin{aligned}
& \left|\frac{\Upsilon\left(\mathfrak{a}_{2}\right)+\Upsilon\left(\mathfrak{a}_{2}+\mathcal{F}_{\rho, \lambda}^{\sigma}\left(\mathfrak{a}_{1}-\mathfrak{a}_{2}\right)\right)}{2}-\frac{1}{\mathcal{F}_{\rho, \lambda}^{\sigma}\left(\mathfrak{a}_{1}-\mathfrak{a}_{2}\right)} \int_{\mathfrak{a}_{2}}^{\mathfrak{a}_{2}+\mathcal{F}_{\rho, \lambda}^{\sigma}\left(\mathfrak{a}_{1}-\mathfrak{a}_{2}\right)} \curlyvee(x) d x\right| \\
& \leq \frac{\left|\mathcal{F}_{\rho, \lambda}^{\sigma}\left(\mathfrak{a}_{1}-\mathfrak{a}_{2}\right)\right|}{8}\left[\left(\frac{\left|\Upsilon^{\prime}\left(\mathfrak{a}_{1}\right)\right|^{q}}{4}+\frac{3\left|\Upsilon^{\prime}\left(\mathfrak{a}_{2}\right)\right|^{q}}{4}\right)^{\frac{1}{q}}+\left(\frac{3\left|\Upsilon^{\prime}\left(\mathfrak{a}_{1}\right)\right|^{q}}{4}+\frac{\left|\Upsilon^{\prime}\left(\mathfrak{a}_{2}\right)\right|^{q}}{4}\right)^{\frac{1}{q}}\right] .
\end{aligned}
$$

Corollary 11 If we put $s=\mathfrak{m}=1$ and $\mathcal{F}_{\rho, \lambda}^{\sigma}\left(\mathfrak{a}_{1}-\mathfrak{a}_{2}\right)=\mathfrak{a}_{1}-\mathfrak{a}_{2}$ in Theorem 14, we get inequality (4.5) in [18].

Note: We pass the some comments regarding comparison on the above estimations of the mentioned lemma. On Lemma 2, we examined Theorem 11 and Theorem 13, in which we used the Hölder and Hölder-İscan inequality. On the comparison, Theorem 13 gives a better result as compared to Theorem 11. Similarly, on Lemma 2, we examined Theorem 12 and Theorem 14, in which we used power mean and improved power mean inequality. On the comparison, Theorem 14 gives a better result as compare to Theorem 12.

\section{Conclusion}

In the development of this paper, some results have been established that generalize, from the definition of Raina integral operator and the use of $s$-type $\mathfrak{m}$-convex function. In particular, those concerning the integral inequality of Hermite-Hadamard. Some algebraic properties are attained in relation to the newly introduced definition. In addition, we described the novel variant of Hermite-Hadamard type inequality in the manner of a generalized $s$-type $\mathfrak{m}$-convex function of Raina type. Our attaining results in the order of lemma can be considered as refinements and remarkable extensions to the new family of generalized convex functions of Raina type. In the future, we hope the results of this paper and the new idea can be extended in different directions like fractional calculus, quantum calculus, time scale calculus, etc. We hope the consequences and techniques of this article will energize and inspire the researcher to explore a more interesting sequel in this area.

\section{Declarations}

\section{Consent for publication}

Not applicable.

\section{Conflicts of interest}

The authors declare that they have no known competing financial interests or personal relationships that could have appeared to influence the work reported in this paper.

\section{Funding}

The authors declare that there is no funding source for the reported research. 


\section{Author's contributions}

M.T.: Conceptualization, Methodology, Software, Writing-Reviewing and Editing. H.A.: Supervision, Investigation, Data Curation, Writing-Original draft preparation. S.K.S.: Visualization, Investigation, Methodology, Software, Writing-Reviewing and Editing. All authors discussed the results and contributed to the final manuscript.

\section{Acknowledgement}

Not applicable.

\section{References}

[1] Hardy, G.H., Little, J.E. and Polya, G. Inequalities. Cambridge, UK. Cambridge University Press. Cambridge mathematical library, (1952).

[2] Kadakal, H. Hermite-Hadamard type inequalities for trigonometrically convex functions. Scientific Studies and Research. Series Mathematic and İnformatics, 28(2), 19-28, (2018).

[3] Niculescu, C.P. and Persson, L.E. Convex functions and their applications.Springer, New York, (2006).

[4] Tariq, M. New Hermite-Hadamard type inequalities via $p$-harmonic exponential type convexity and applications. Universal Journal of Mathematics and Applications, 4(2), 59-69, (2021).

[5] Özcan, S. and İşcan, İ. Some new Hermite-Hadamard type integral inequalities for the $s$-convex functions and theirs applications. Journal of Inequalities and Applications, 2019(201), 1-14, (2019).

[6] Tariq, M., Nasir, J., Sahoo, S.K. and Mallah, A.A. A note on some Ostrowski type inequalities via generalized exponentially convex function. Journal of Mathematical Analysis and Modeling, 2(2), 1-15, (2021).

[7] Khan, M.A., Chu, Y.M., Khan, T.U. and Khan, J. Some new inequalities of Hermite-Hadamard type for s-convex functions with applications. Open Math, 15(1), 1414-1430, (2017).

[8] Butt, S.I., Tariq, M., Aslam, A., Ahmad, H. and Nofel, T.H. Hermite-Hadamard type inequalities via generalized harmonic exponential convexity. Journal of Function Spaces, 1-12, (2021).

[9] Xi, B.Y. and Qi, F. Some integral inequalities of Hermite-Hadamard type for convex functions with applications to means. Journal of Function Spaces, 1-14, (2012).

[10] Butt, S.I., Kashuri, A., Tariq, M., Nasir, J., Aslam, A. and Geo, W. Hermite-Hadamard-type inequalities via $n$-polynomial exponential-type convexity and their applications. Advances in Difference Equations, 508, (2020).

[11] Butt, S.I., Kashuri, A., Tariq, M., Nasir, J., Aslam, A. and Geo, W. n-polynomial exponential-type p-convex function with some related inequalities and their application. Heliyon, 6(11), (2020).

[12] Hadamard, J. Étude sur les propriétés des fonctions entiéres en particulier d'une fonction considéréé par Riemann. Journal of Mathematics. Pures. Appl, 58, 171-215, (1893).

[13] Raina, R.K. On generalized Wright's hypergeometric functions and fractional calculus operators. East Asian Mathematical Journal, 21(2), 191-203, (2005).

[14] Cortez, M.J.V., Liko, R., Kashuri, A. and Hernández, J.E.H. New quantum estimates of trapezium-type inequalities for generalized $\phi$-convex functions. Mathematics, 7(11), 1047-1066, (2019).

[15] Cortez, M.J.V., Kashuri, A. and Hernández, J.E.H. Trapezium-type inequalities for Raina's fractional integrals operator using generalized convex functions.Symmetry, 12(6), 1034, (2020).

[16] Rashid, S., İşcan, İ., Baleanu, D. and Chu, Y.M. Generation of new fractional inequalities via n-polynomials s-type convexity with applications. Advances in Difference Equations, Article ID 264

[17] Barani, A., Ghazanfari, A.G. and Dragomir, S.S. Hermite-Hadamard inequality for functions whose derivatives absolute values are preinvex. Journal of Inequalities and Applications, 2012(1), 1-9, (2012).

[18] Toplu, T., Kadakal, M. and İşcan, İ. On n-polynomial convexity and some related inequalities. AIMS Math, 5(2), 1304-1318, (2020).

Mathematical Modelling and Numerical Simulation with Applications (MMNSA) (http://www.mmnsa.org)

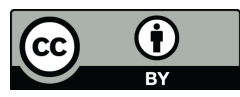

Copyright: ( $) 2021$ by the authors. This work is licensed under a Creative Commons Attribution 4.0 (CC BY) International License. The authors retain ownership of the copyright for their article, but they allow anyone to download, reuse, reprint, modify, distribute, and/or copy articles in MMNSA, so long as the original authors and source are credited. To see the complete license contents, please visit (http://creativecommons.org/licenses/by/4.0/). 